\title{
Diabetic Retinopathy in the Spontaneously Diabetic Torii Rat: Pathogenetic Mechanisms and Preventive Efficacy of Inhibiting the Urokinase-Type Plasminogen Activator Receptor System
}

\author{
Maurizio Cammalleri, ${ }^{1}$ Massimo Dal Monte, ${ }^{1}$ Filippo Locri, ${ }^{1}$ Stefania Marsili, ${ }^{1}$ Liliana Lista, ${ }^{2}$ \\ Mario De Rosa, ${ }^{3}$ Vincenzo Pavone, ${ }^{2}$ Dario Rusciano, ${ }^{4}$ and Paola Bagnoli ${ }^{1}$ \\ ${ }^{1}$ Department of Biology, University of Pisa, Via San Zeno 31, 56127 Pisa, Italy \\ ${ }^{2}$ Department of Biology, University of Napoli Federico II, Complesso Universitario Monte Sant'Angelo, Via Cinthia, Edificio 7 , \\ 80126 Napoli, Italy \\ ${ }^{3}$ Department of Experimental Medicine, Second University of Napoli, Via Santa Maria di Costantinopoli 16, 80138 Napoli, Italy \\ ${ }^{4}$ Sooft Fidia Pharma, Contrada Molino 17, 63833 Montegiorgio, Italy
}

Correspondence should be addressed to Massimo Dal Monte; massimo.dalmonte@unipi.it

and Paola Bagnoli; paola.bagnoli@unipi.it

Received 24 August 2017; Revised 20 October 2017; Accepted 13 November 2017; Published 31 December 2017

Academic Editor: Ilaria Campesi

Copyright ( 2017 Maurizio Cammalleri et al. This is an open access article distributed under the Creative Commons Attribution License, which permits unrestricted use, distribution, and reproduction in any medium, provided the original work is properly cited.

The spontaneously diabetic Torii (SDT) rat is of increasing preclinical interest because of its similarities to human type 2 diabetic retinopathy (DR). The system formed by urokinase-type plasminogen activator (uPA) and its receptor (uPAR) is a player in blood-retinal barrier (BRB) breakdown in DR. Here, we investigated whether in SDT rats, preventive administration of UPARANT, an inhibitor of the uPAR pathway, counteracts the retinal impairment in response to chronic hyperglycemia. Electroretinogram (ERG) monitoring was followed over time. Fluorescein-dextran microscopy, CD31 immunohistochemistry, quantitative PCR, ELISA, Evans blue perfusion, and Western blot were also used. UPARANT prevented ERG dysfunction, upregulation of vascular endothelial growth factor and fibroblast growth factor-2, BRB leakage, gliosis, and retinal cell death. The mechanisms underlying UPARANT benefits were studied comparing them with the acute streptozotocin (STZ) model in which UPARANT is known to inhibit DR signs. In SDT rats, but not in the STZ model, UPARANT downregulated the expression of UPAR and its membrane partners. In both models, UPARANT reduced the levels of transcription factors coupled to inflammation or inflammatory factors themselves. These findings may help to establish the uPAR system as putative target for the development of novel drugs that may prevent type $2 \mathrm{DR}$.

\section{Introduction}

Diabetic retinopathy (DR) is a serious complication of diabetes that accounts for the large majority of cases of adult blindness in working age population. Considering that type 1 diabetes accounts for less than $10 \%$ of all cases of diabetes [1], in most instances, DR turns out to be a disease associated to type 2 diabetes.

In type 1 diabetes, its early onset allows a precocious detection of the disease thus permitting a better control of
$\mathrm{DR}$, whereas, in type 2 diabetes, the late onset of DR delays its therapeutical treatments that are usually given when the disease has become vision-threating and are not always successful in restoring vision loss. Therefore, current therapeutic strategies show an unmet clinical need for therapies to prevent the occurrence and/or progression of DR in type 2 diabetes.

The streptozotocin- (STZ-) induced rodent model is an acknowledged model of type 1 diabetes in which DR is established early after diabetes onset, and its features resemble 
those of the early stages of DR in patients [2]. On the other hand, the literature concerning animal models of type 2 diabetes is more sparse and most developing therapies against type 2 DR have been extrapolated from the STZ model [3]. One of the best models mimicking at least in part the pathologic signs of type 2 diabetes is the spontaneously diabetic Torii (SDT) rat. The SDT rat is an inbred rat strain isolated from an outbred colony of Sprague-Dawley (SD) rats and is characterized by late diabetes onset followed by DR. Indeed, male SDT rats spontaneously develop hyperglycemia after 20 weeks of age, becoming diabetic without obesity [4]. Despite the chronic severe hyperglycemia, SDT rats survive for a long time without insulin treatment thus rendering them suitable for preventive drug efficacy studies. SDT rats are characterized by DR that becomes established at about 20 weeks after diabetes onset and is followed by severe ocular complications including upregulated expression of vascular endothelial growth factor (VEGF), structural impairment of the neuroretina and gliosis, blood-retinal barrier leakage, and reduced electroretinogram (ERG) amplitude [5].

Recently, the system formed by urokinase-type plasminogen activator (uPA) and its receptor (UPAR) has been receiving particular attention as it is likely to be a major player in BRB breakdown in the presence of DR. This system, in fact, is upregulated in response to high glucose [6-8]. Accordingly, BRB leakage can be prevented by deletion of the UPAR gene or administration of A6, a peptide that inhibits the interaction between uPA and uPAR $[6,7]$. Among peptide inhibitors of the uPAR system, a tetrapeptide named UPARANT, designed to mimic the amino acid sequence through which uPAR binds its interactors in the cellular membrane, including the formyl peptide receptors (FPRs), displays resistance to enzymatic digestion, high stability in blood and plasma, and optimal effectiveness as a uPAR inhibitor [9]. UPARANT has been shown to play antiangiogenic and anti-inflammatory actions in different models of neovascular ocular pathologies $[10,11]$. In the STZ rat, UPARANT has been shown to act in a therapeutic regimen by recovering the pathological signs of early DR [12], but the short-lasting duration of the disease renders this model unfit to determine possible preventive effects of the drug. Demonstrating preventive efficacy of UPARANT in models of long-lasting DR would help to establishing the uPAR system as putative target for the development of novel therapies. In the present study, we used the SDT rat as a model of late onset, long-lasting DR to investigate whether repeated systemic administration of UPARANT might prevent retinal impairment in response to persisting hyperglycemia.

\section{Materials and Methods}

2.1. Reagents. UPARANT was synthesized as previously described [9]. The monoclonal mouse anti-rat antibody directed against CD31 was from BD Pharmingen (San Diego, CA, USA). The AllPrep RNA/Protein Kit was purchased from Qiagen (Valencia, CA, USA). The master mix (SsoAdvanced Universal SYBR Green Supermix), the polyvinylidene difluoride membranes, and the enhanced chemiluminescence reagent were from Bio-Rad Laboratories (Hercules, CA, USA). Primers were obtained from ThermoFisher (Waltham, MA, USA). The ELISA kits were from R\&D Systems (Minneapolis, MN, USA). The goat polyclonal antibody directed to claudin-1 (sc-17658), zonula occludens-1 (ZO-1; sc-8146), or FPR1 (sc-13198), the rabbit polyclonal antibodies directed to claudin-5 (sc-28670), glial fibrillary acidic protein (GFAP; sc-9065), uPAR (sc-10815), FPR2 (sc-66901), FPR3 (sc-66899), the p65 subunit of the nuclear factor kappa-light-chain-enhancer of activated $\mathrm{B}$ cells (NF- $\kappa \mathrm{B}$ p65; sc-372), NF- $\kappa \mathrm{B}$ p65 phosphorylated at $\operatorname{Ser}^{276}$ (pNF- $\kappa \mathrm{B}\left(\mathrm{Ser}^{276}\right)$; sc-101749), or signal transducer and activator of transcription 3 (STAT3; sc-482), the mouse monoclonal antibody directed to STAT3 phosphorylated at $\mathrm{Tyr}^{705}$ (pSTAT3 $\left(\mathrm{Tyr}^{705}\right.$ ); sc-8059), and the mouse antirabbit and the rabbit anti-goat horseradish peroxidaselabeled secondary antibodies were purchased from Santa Cruz Biotechnologies (Santa Cruz, CA, USA). The rabbit monoclonal antibody directed to the active (cleaved) form of caspase-3 (number 9664) is from Cell Signaling Technology (Danvers, MA, USA). All other chemicals were purchased from Sigma-Aldrich (St. Louis, MO, USA).

2.2. Animals and UPARANT Treatment. Male SDT rats $(n=25)$ were purchased from CLEA Japan Inc. (Tokyo, Japan). All animal experiments were conducted in accordance with the guidelines for care and use of experimental animals according to the European Communities Council (2010/63/UE L 27620/10/2010), the Italian law (DL: 04.03.2014, number 26), and the ARVO Statement for the Use of Animals in Ophthalmic and Vision Research. Experimental procedures were approved by the Ethical Committee in Animal Experiments of the University of Pisa. All efforts were made to minimize suffering and the numbers of animals used. In this respect, the number of animals used here was limited by the rules of the Ethical Committee due to the limited space to stock animals with long-lasting diseases in the facility. Age-matched SD rats (150-200 g) were obtained from Charles River Laboratories, Italia (Calco, Italy). Twelve of them were used as nondiabetic controls. Additional 9 SD rats were treated with a single intraperitoneal injection of $65 \mathrm{mg} / \mathrm{kg} \mathrm{STZ}$, dissolved in citrate buffer ( $\mathrm{pH} 4.5)$, to induce diabetes. Blood glucose was measured by tail sampling using a OneTouch Ultra glucometer (LifeScan Inc., Milpitas, CA, USA). SDT and STZ rats were confirmed to be diabetic based on a nonfasting blood glucose values $\geq 350 \mathrm{mg} / \mathrm{dL}$. Weight and blood glucose of nondiabetic SD or SDT rats are reported in Figures 1(a) and 1(b). No differences in weight and blood glucose were observed in SDT rats untreated, vehicle-treated, or UPARANTtreated. UPARANT was dissolved in phosphate-buffered saline (PBS) and subcutaneously administered. In SDT rats, treatments were initiated 7 weeks after diabetes onset, a time corresponding to the thirteen weeks before the occurrence of significant ERG dysfunctions. Of the SDT rats, 9 rats received UPARANT at $7 \mathrm{mg} / \mathrm{kg}$ ( 3 times a week), 8 rats received PBS, and 8 rats were left untreated. In STZ rats, treatments were initiated 4 weeks after diabetes onset, a time corresponding to the onset of dysfunctional ERG [12]. Of the 


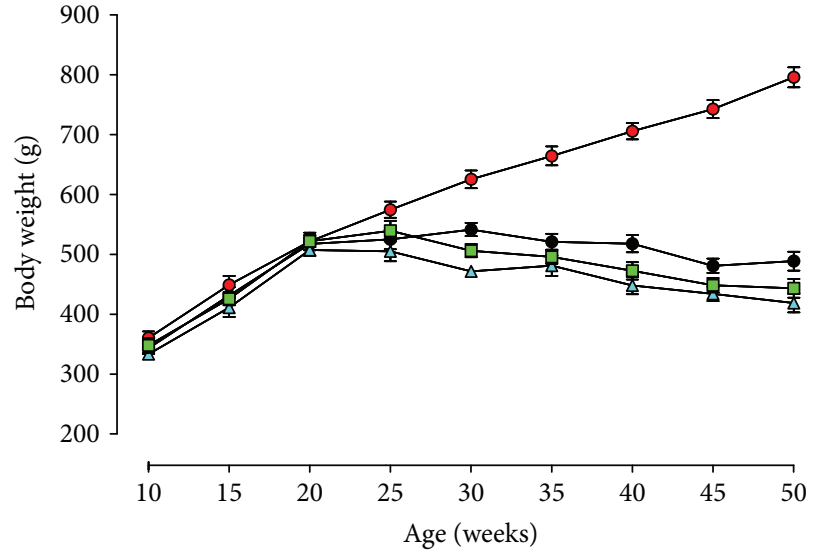

(a)

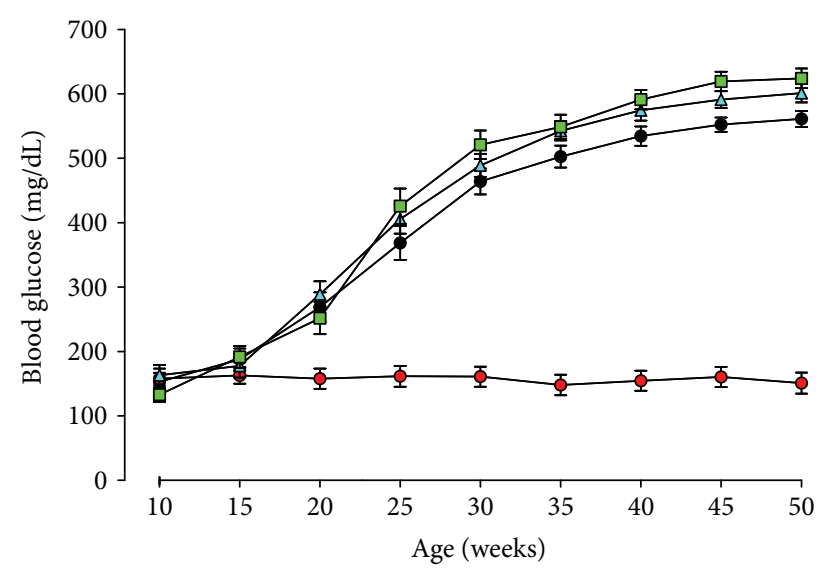

(b)

Figure 1: Body weight and blood glucose levels in SD and SDT rats. Mean body weight (a) and blood glucose levels (b) in SD $(n=9$, red circles) and in SDT rats either untreated ( $n=8$, black circles), vehicle-treated ( $n=8$, light blue triangles), or UPARANT-treated ( $n=9$, green squares).

STZ rats, 3 rats received UPARANT $(8 \mathrm{mg} / \mathrm{kg}$ daily for 5 days, according to [12]), 3 rats received PBS, and 3 rats were left untreated. In all experiments, rats were anesthetized with an intraperitoneal injection of pentobarbital $(30 \mathrm{mg} / \mathrm{kg})$.

2.3. Electroretinographic Recording. In all SDT rats, retinal function was monitored longitudinally with scotopic fullfield ERG. Before ERG testing, rats were dark adapted overnight. After anesthesia, rat pupils were dilated with $0.5 \%$ atropine and the cornea was intermittently irrigated with saline solution to prevent clouding of the ocular media. A heating pad was used to keep the body temperature at $38^{\circ} \mathrm{C}$. The ERG responses were recorded from both eyes through silver/silver chloride corneal electrodes and a forehead reference electrode. A ground electrode was placed on the tail. Scotopic ERG responses, which primarily measure rod function, were triggered by flashes of different light intensities ranging from -3.4 to $1 \log \mathrm{cd}-\mathrm{s} / \mathrm{m}^{2}$ generated through a Ganzfeld stimulator (Biomedica Mangoni, Pisa, Italy). The electrodes were connected to a two-channel amplifier. Signals were amplified at 1000 gain and bandpass filtered between 0.2 and $500 \mathrm{~Hz}$ before being digitized at $5 \mathrm{kHz}$ rate with a data acquisition device (Biomedica Mangoni). The ERG waveforms were examined primarily for amplitude information (i.e., the size of the a- and b-waves) and the data were graphed to determine any gross changes in the intensityresponse function for that eye. Data were pooled and reported as mean amplitude \pm SEM (in $\mu \mathrm{V}$ ). Intensityresponse functions of the $b$-wave were fit to a modified Naka-Rushton equation [13].

$$
V(I)=\frac{V 0+(V \max \mathrm{In})}{(\operatorname{In}+k n)}
$$

where $V$ is the amplitude of the b-wave (in $\mu \mathrm{V}$ ), $I$ is the stimulus intensity (in $\log \mathrm{cd}-\mathrm{s} / \mathrm{m}^{2}$ ), $V 0$ is the nonzero baseline effect, $V \max$ is the saturated amplitude of the b-wave (in $\mu \mathrm{V}$ ), $k$ is the stimulus intensity that evokes a b-wave of half-maximum amplitude (in $\log \mathrm{cd}-\mathrm{s} / \mathrm{m}^{2}$ ), and $n$, which was constrained to unity, is a dimensionless constant controlling the slope of the function and represents the degree of heterogeneity of retinal sensitivity.

2.4. Fluorescein-Dextran Microscopy. Fluorescein-conjugated dextran perfusion of retinal vessels was performed in anesthetized animals using (i) 4 retinas from untreated or vehicle-treated SDT rats and (ii) 6 retinas from UPARANT-treated SDT or control SD rats. After rats were anesthetized, a median sternotomy was performed. The left ventricle was perfused with $2 \mathrm{~mL}$ of $25 \mathrm{mg} / \mathrm{mL}$ fluoresceinconjugated dextran in $0.15 \mathrm{M}$ phosphate buffer (PB). Ten minutes after perfusion, the eyes were enucleated for fluorescein microscopy. The retinas were dissected, and flat mounts were obtained and mounted in antifade medium (Vectashield; Vector Laboratories, Burlingame, CA), vitreous side up under coverslips. Whole mounts were viewed by fluorescence microscopy (Ni-E; Nikon Europe, Amsterdam, The Netherlands). Images were acquired (DS-Filc camera; Nikon Europe), and an image-editing software (Adobe Photoshop CS3; Adobe System Inc., Mountain View, CA, USA) was used to create whole retina montages.

2.5. CD-31 Immunohistochemistry. In whole retinas from 3 different SDT or control SD rats, the retinal vasculature was visualized using antibodies directed to CD31. Dissected retinas were immersion fixed for 1.5 hours in $4 \%$ paraformaldehyde in $0.1 \mathrm{M} \mathrm{PB}$ at $4^{\circ} \mathrm{C}$. They were then transferred to $25 \%$ sucrose in $0.1 \mathrm{M} \mathrm{PB}$ and stored at $4^{\circ} \mathrm{C}$. The whole mounts were freeze-thawed and incubated for 72 hours at $4^{\circ} \mathrm{C}$ in the CD31 antibody (1:50 in $0.1 \mathrm{M} \mathrm{PB}$ containing $0.5 \%$ Triton $\mathrm{X}-100)$. They were then incubated for 48 hours at $4^{\circ} \mathrm{C}$ with AlexaFluor 488-conjugated secondary antibody (1:200 in $0.1 \mathrm{M} \mathrm{PB}$ containing $0.5 \%$ Triton X-100). Finally, the whole mounts were rinsed in $0.1 \mathrm{M} \mathrm{PB}$, mounted on gelatin-coated glass slides, and coverslipped with a $0.1 \mathrm{M}$ PB-glycerin mixture. Images were acquired with a microscope equipped with epifluorescence (Ni-E; Nikon Europe, Amsterdam, The Netherlands) through a digital camera 
TABLE 1: Sequences of primer sets used for qPCR experiments.

\begin{tabular}{|c|c|c|}
\hline \multirow{2}{*}{ Gene } & \multicolumn{2}{|c|}{ Primer sequence $\left(5^{\prime} \rightarrow 3^{\prime}\right)$} \\
\hline & Forward primer & Reverse primer \\
\hline VEGF & TGTGAGCCTTGTTCAGAGCGG & ACTCAAGCTGCCTCGCCTTGC \\
\hline$F G F-2$ & GCGGCTCTACTGCAAGA & CGTCCATCTTCCTTCATAGC \\
\hline Claudin-1 & GTTTCATCCTGGCTTCGCTG & CTTTGCGAAACGCAGGACAT \\
\hline Claudin-5 & TACTCAGCACCAAGGCGAAC & TTCCCACATCGGTCTTTCCG \\
\hline $\mathrm{ZO}-1$ & AGTCTCGGAAAAGTGCCAGG & GGGCACCATACCAACCATCA \\
\hline GFAP & TGACGCCTCCACTCCCTGCC & CATCTCCGCACGCTCGCTGG \\
\hline Caspase-3 & ССТTTCСTCTCCACCGTAGA & AGATGCCACCTCTCCTTTCC \\
\hline$u P A R$ & TTGGATGTTCCTACGAAGAGACG & GTAACTCCGGTTTCCCAGCA \\
\hline FPR1 & GTTTCCGCATGAAACGCACT & CATGACCAGGCTGACGATGT \\
\hline FPR2 & GCTTCACAATGCCCATGTCC & ACTCGTAAGGGACGACTGGA \\
\hline FPR3 & TCCCTTTCAACTGGTTGCCC & GCCAATGAGTTGGTTGGCATA \\
\hline$T N F-\alpha$ & СССТСАСАСТСАGTCATCTTCT & GTCACGACGTGGGCTACAG \\
\hline$I L-1 \beta$ & САССТСТСAAGCAGAGCACAG & GGGTTCCATGGTGAAGTCAAC \\
\hline$I L-6$ & TCСТАССССААСТTССАAТGСТС & TTGGATGGTCTTGGTCCTTAGCC \\
\hline Rpl13a & GGATCCCTCCACCCTATGACA & CTGGTACTTCCACCCGACCTC \\
\hline Hprt & CTCATGGACTGATTATGGACAGGAC & GCAGGTCAGCAAAGAACTTATAGCC \\
\hline
\end{tabular}

(DS-Filc; Nikon Europe). An image-editing software (Adobe Photoshop CS3; Adobe System Inc., Mountain View, CA, USA) was used to create whole retina montages on which the subsequent quantification was performed.

2.6. Quantitative Real-Time PCR. Quantitative real-time PCR (qPCR) experiments were performed using 3 independent samples, each containing 1 retina, for each experimental condition. Total RNA and proteins were extracted using an isolation kit (AllPrep RNA/Protein Kit; Qiagen Inc.). Purified RNA was resuspended in RNase-free water and quantified using a fluorometer (Qubit; Invitrogen, Carlsbad, CA, USA). First-strand cDNA was generated from $1 \mu \mathrm{g}$ of total RNA (QuantiTect Reverse Transcription Kit; Qiagen). Real-time PCR amplification was performed with a kit (SsoAdvanced Universal SYBR Green Supermix; Bio-Rad Laboratories) on a CFX Connect Real-Time PCR Detection System and software CFX manager (Bio-Rad Laboratories). qPCR primer sets for VEGF, fibroblast growth factor-2 (FGF-2), claudin-1 and claudin-5, ZO-1, GFAP, caspase-3, uPAR, FPRs, tumor necrosis factor- $\alpha$ (TNF- $\alpha$ ), interleukin(IL-) $1 \beta$, and IL- 6 were chosen to hybridize to unique regions of the appropriate gene sequence (see Table 1 for a complete list of assayed genes and primers). Amplification efficiency was close to $100 \%$ for each primer pair (Opticon Monitor 3 software; Bio-Rad Laboratories). Target genes were assayed concurrently with Rpl13a and Hprt, genes encoding for ribosomal protein L13A and hypoxanthine guanine phosphoribosyl transferase, respectively. Samples were compared using the relative threshold cycle ( $\mathrm{Ct}$ method). The increase or decrease (fold change) was determined relative to control SD rats after normalization to Rpl13a and Hprt. All reactions were performed in triplicate. After statistical analysis, the data from the different experiments were plotted and averaged in the same graph.
2.7. Enzyme-Linked Immunosorbent Assay. Quantification of VEGF, FGF-2, TNF- $\alpha$, IL- $1 \beta$, and IL-6 protein levels was performed with commercially available kits using proteins purified as described above. Protein concentration was determined with a fluorometer (Qubit; Invitrogen). ELISA plates were evaluated spectrophotometrically (Microplate Reader 680 XR; Bio-Rad Laboratories) according to the manufacturers' instructions. Data were expressed as nanograms or picograms of targets per milligram of protein. All experiments were performed in duplicate.

2.8. Measurement of Retinal Vascular Leakage by Evans Blue Dye. Diabetes-induced leakage was evaluated by assessment of Evans blue dye extravasation using 3 retinas from 3 different control SD and untreated, vehicle-treated, or UPARANT-treated SDT rats. Anesthetized rats were injected with Evans blue dye $(0.5 \%$ in PBS) into the left ventricle. For quantitative evaluation of BRB leakage, the animals were euthanized, the eyes were enucleated, and the retinas isolated and weighted. The Evans blue dye was extracted with formamide overnight at $65^{\circ} \mathrm{C}$ and read at $620 \mathrm{~nm}$ using a plate reader (Microplate Reader $680 \mathrm{XR}$; Bio-Rad Laboratories). For qualitative evaluation of outer BRB leakage, enucleated eyes were fixed in $4 \%$ paraformaldehyde. The retinas were flat mounted and examined with a fluorescence microscope (Ni-E; Nikon Europe, Amsterdam, The Netherlands), and images were acquired (DS-Filc camera; Nikon-Europe).

2.9. Western Blot Analysis. Proteins were purified as described above and supplemented with protease and phosphatase inhibitor cocktails. Aliquots of each sample containing equal amounts of protein $(30 \mu \mathrm{g})$ were subjected to SDSPAGE. The gels were transblotted onto PVDF membranes that were blocked in $3 \%$ skim milk and incubated 
overnight at $4^{\circ} \mathrm{C}$ with primary antibodies. The goat polyclonal antibodies directed to claudin-1, ZO-1, or FPR1, or the rabbit polyclonal antibodies directed to claudin-5, GFAP, uPAR, FPR2, FPR3, NF- $\kappa$ B p65, pNF- $\kappa$ B p65 $\left(\mathrm{Ser}^{276}\right)$, or STAT3, or the monoclonal antibody directed to the active form of caspase- 3 , or the mouse monoclonal antibody directed to PSTAT3 $\left(\mathrm{Tyr}^{705}\right)$ were used. The antibodies were used at $1: 200$ dilutions with the exception of the antibodies directed to either GFAP $(1: 300)$ or the active form of caspase-3 $(1: 1000)$. The same membranes were reblotted with the mouse monoclonal antibody directed to $\beta$-actin $(1: 2500)$ used as the loading control. Finally, PVDF membranes were incubated for $1 \mathrm{~h}$ at room temperature with mouse anti-rabbit $(1: 5000)$, rabbit anti-goat $(1: 5000)$, or rabbit anti-mouse $(1: 25,000)$ horseradish peroxidaselabeled secondary antibodies, as appropriate, and developed with the enhanced chemiluminescence reagent. Images were acquired using the Chemidoc $\mathrm{XRS}^{+}$(Bio-Rad Laboratories). The optical density (OD) of the bands was evaluated with the Image Lab 3.0 software (Bio-Rad Laboratories). The data were normalized to the level of $\beta$-actin, NF- $\kappa \mathrm{B}$ p 65 , or STAT3 as appropriate. All experiments were run in duplicate. After statistics, data were averaged and plotted in the same graph.

2.10. Statistical Analysis. Statistical significance was evaluated using one-way analysis of variance (ANOVA) followed by Newman-Keuls' multiple comparison posttest or two-way ANOVA followed by Bonferroni's multiple comparison posttest as appropriate. Despite the limited number of samples per group, a parametric analysis was performed as the data inside each of the samples were normally distributed. The results were expressed as mean \pm SEM of the indicated $n$ values (Prism 5.03; GraphPad software, San Diego, CA, USA). Differences with $P<0.05$ were considered significant. After the data were collected, post hoc power analysis was conducted using the software $\mathrm{G}^{*}$ Power 3.0.10 in order to determine the statistical power of the results obtained in experiments with a small number of samples per group. A power value of 0.80 was considered the required minimum value to reject the null hypothesis.

\section{Results}

3.1. UPARANT Prevents ERG Dysfunction. In a longitudinal study, ERG was repeatedly recorded at different times in each SDT rat from the diabetes onset until the twenty-fifth week, before the development of severe lens opacity which might have prevented ERG evaluation. The representative recordings in Figure 2(a) show mixed a- and b-waves recorded at light intensities of $1 \log \mathrm{cd}-\mathrm{s} / \mathrm{m}^{2}$ in untreated, vehicle-treated, or UPARANT-treated rats. It can be noticed that starting from the twentieth week after diabetes onset, both in untreated and in vehicle-treated SDT rats, the ERG amplitude was decreased, in line with previous studies [14], while in UPARANT-treated SDT rats, the ERG amplitude remained unaltered over time. The diagrams in Figures $2(\mathrm{~b})$ and 2(c) show amplitude reduction of both the a-wave (at light intensities ranging from -0.3 to $1 \log \mathrm{cd}-\mathrm{s} / \mathrm{m}^{2}$;
$P<0.001$ ) and the b-wave (at light intensities ranging from -2.8 to $\left.1 \log \mathrm{cd}-\mathrm{s} / \mathrm{m}^{2} ; P<0.001\right)$ in SDT rats, untreated, or vehicle-treated, in respect to $\mathrm{SD}$ control animals. In UPARANT-treated rats, the amplitudes of the a- and bwaves did not significantly differ from those measured in $\mathrm{SD}$ rats. Intensity-response functions of the b-wave as evaluated by a fitted Naka-Rushton equation demonstrated that the b-wave amplitude $(V \max )$ and the retinal sensitivity $(k)$ in untreated or vehicle-treated SDT rats were significantly lower than in SD rats, whereas UPARANT treatment prevented the reduction of $V \max$ and $k$ (Table 2).

3.2. UPARANT Prevents Retinal Leakage. Twenty-five weeks after diabetes onset, perfusion with fluorescein demonstrated that, in comparison with SD rats (Figure 3(a)), untreated SDT rats showed abnormal retinal vasodilatation together with severe fluorescein leakage (Figure 3(b)) while the leakage is prevented by UPARANT administration (Figure 3(c)). Defects comparable to those in untreated SDT rats were observed in vehicle-treated SDT rats (not shown). The retinal microvascular abnormalities evidenced by fluorescein microscopy were not accompanied by an altered pattern of retinal vasculature in the superficial vascular plexus (Figures 4(a) and 4(b)). Analysis of transcripts and proteins demonstrated upregulated levels of both VEGF and FGF2 that were prevented by UPARANT treatment (Figures 5(a)-5(d)). Fluorescein leakage was confirmed by the extravasation of Evans blue, a dye that binds to plasma proteins, and by dysregulated levels of transcripts of $\mathrm{BRB}$ markers. As compared to SD controls (Figure 6(a)), untreated (Figure 6(b)) or vehicle-treated (Figure 6(c)) SDT rats showed a clear extravasation of Evans blue that, on the contrary, was not observed in UPARANT-treated rats (Figure 6(d)). The quantitative analysis (Figure 6(e)) showed that in untreated or vehicle-treated SDT rats, Evans blue dye leakage was increased by approximately 2.2 -fold with respect to SD rats $(P<0.001)$, while UPARANT treatment prevented this increase. Levels of transcripts and proteins of $\mathrm{BRB}$ markers including the transmembrane components of the interendothelial tight junctions claudin-1, claudin-5, and ZO-1 were concomitantly decreased by about 2 -fold $(P<0.001)$ at the transcript (Figures $7(\mathrm{a}), 7(\mathrm{c})$, and $7(\mathrm{e}))$ and the protein (Figures 7(b), 7(d), and 7(f)) level. UPARANT administration prevented the dysregulation of BRB markers with transcript and protein levels that did not differ significantly from those measured in SD rats.

3.3. UPARANT Prevents Retinal Damage. Twenty-five weeks after diabetes onset, qPCR and Western blot demonstrated that, in comparison with SD rats, untreated SDT rats showed increased GFAP expression (Figures 8(a) and 8(b)) presumably coupled to gliotic Müller cells together with increased retinal levels of caspase-3 (Figures 8(c) and 8(d)), a reliable marker of apoptosis-induced retinal neurodegeneration [15]. Upregulated levels of both GFAP and caspase-3 were prevented by UPARANT treatment.

3.4. UPARANT Prevents the Upregulation of the UPAR/FPR System and Its Downstream Effectors. Whether UPARANT 


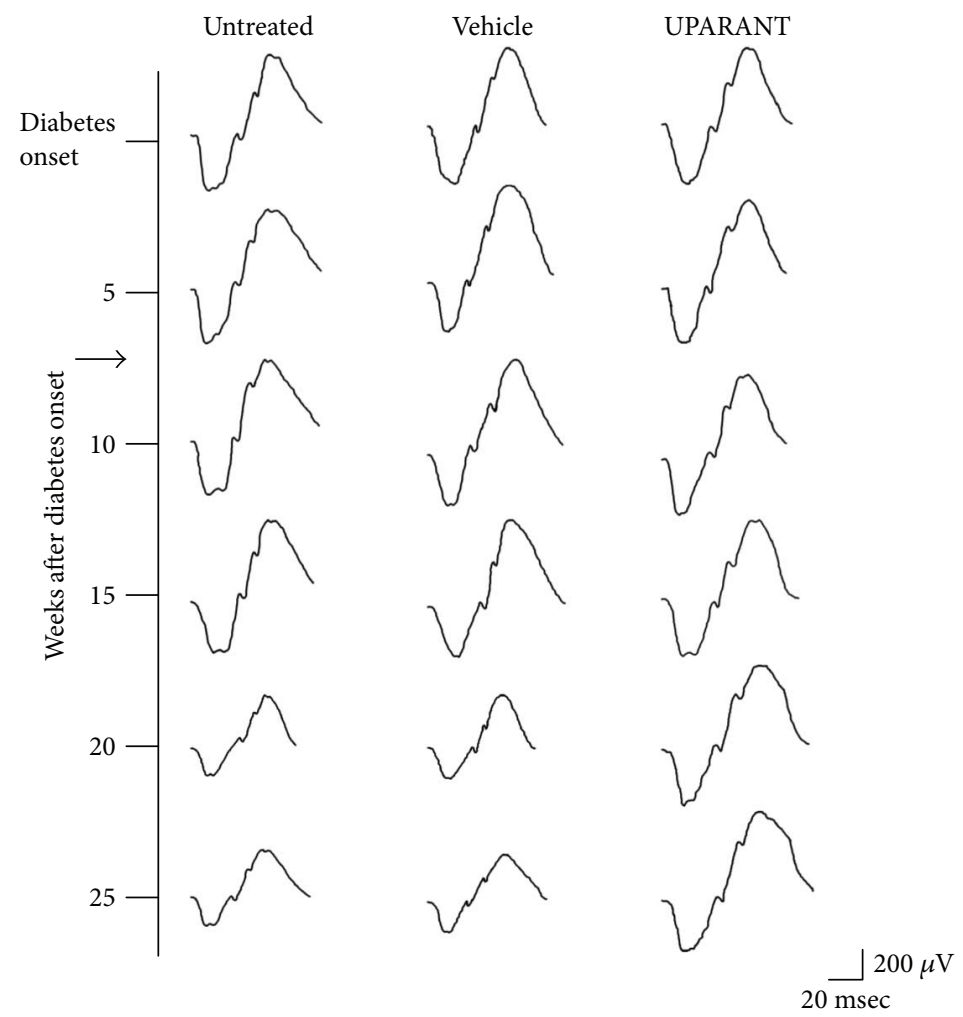

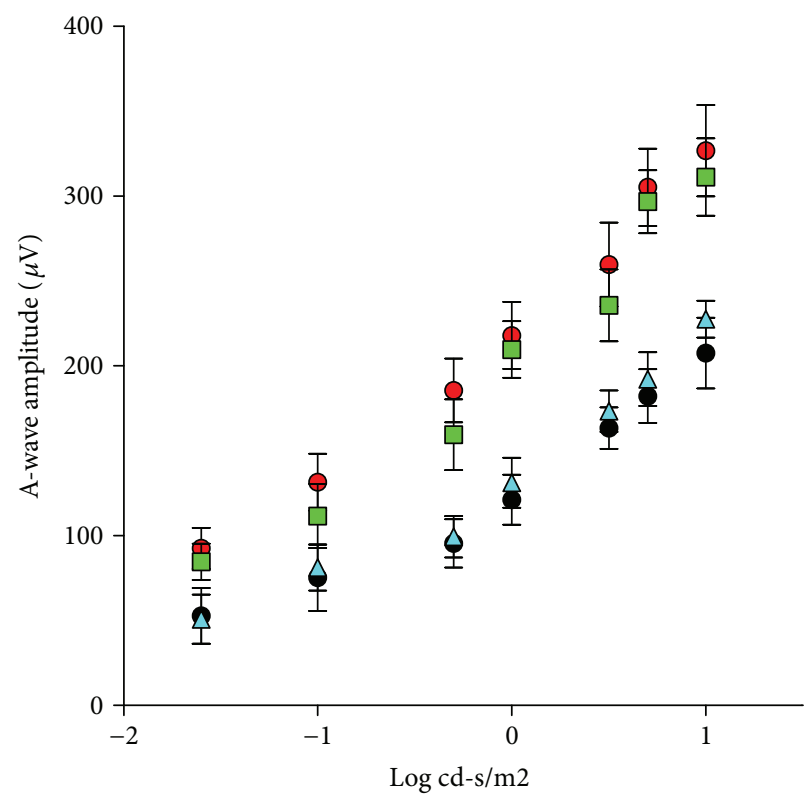

(b)

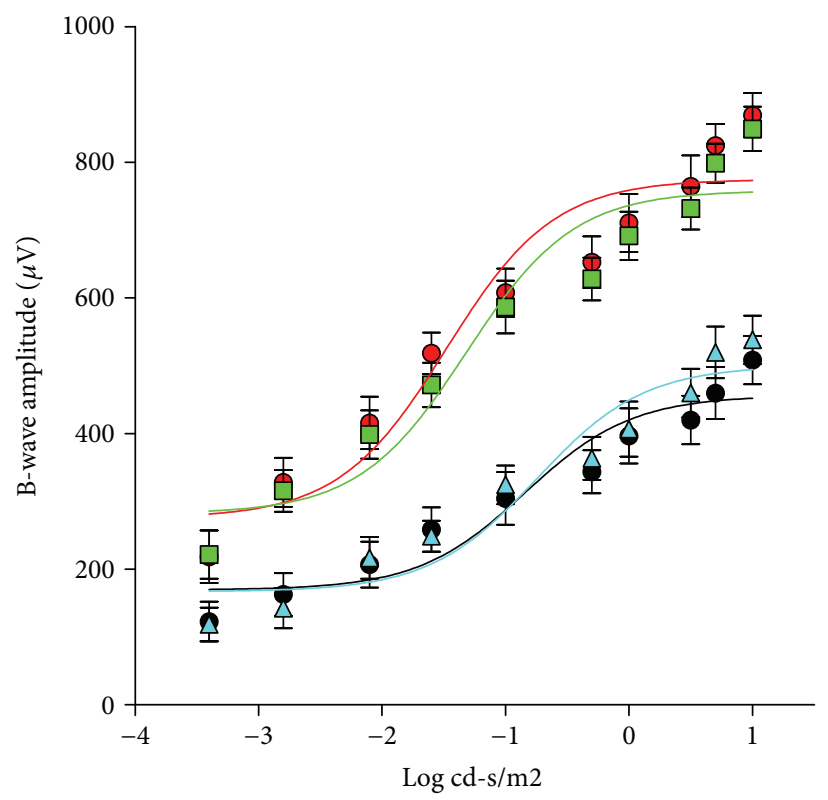

(c)

FIgURE 2: Effects of UPARANT on ERG responses. (a) Schematic representation of longitudinal ERG monitoring at different times after diabetes onset (corresponding to 20 weeks of age). The arrow indicates the beginning of the treatment ( $7 \mathrm{mg} / \mathrm{kg}$ UPARANT or vehicle, given subcutaneously 3 times a week for 19 weeks). The treatment was initiated at 7 weeks after diabetes onset. (b, c) Scotopic a-wave (b) and b-wave (c) amplitudes plotted as a function of increasing light intensity in control SD rats (red circles and red line) and in SDT rats, untreated (black circles and black line), vehicle-treated (light blue triangles and light blue line), or treated with subcutaneous UPARANT (green squares and green line). In respect to SD rats, both a-wave and b-wave amplitude was reduced in SDT rats, untreated or vehicletreated. In UPARANT-treated rats, the amplitudes of the a- and b-waves did not significantly differ from those measured in SD rats (twoway ANOVA followed by Bonferroni's multiple comparison posttest). Each point represents the mean \pm SEM of data from 8 (untreated and vehicle-treated SDT) or 9 (SD controls and UPARANT-treated SDT) rats. 
TABle 2: Parameters obtained from b-wave amplitude using the Naka-Rushton function.

\begin{tabular}{lcccc}
\hline & SD rats & Untreated SDT rats & Vehicle-treated SDT rats & UPARANT-treated SDT rats \\
\hline$V \max (\mu \mathrm{V})$ & $774.80 \pm 20.80$ & $456.20 \pm 21.62^{*}$ & $500.00 \pm 21.96^{*}$ & $758.20 \pm 19.83$ \\
$k\left(\log \mathrm{cd}-\mathrm{s} / \mathrm{m}^{2}\right)$ & $-1.47 \pm 0.15$ & $-0.84 \pm 0.22^{*}$ & $-0.74 \pm 0.18^{*}$ & $-1.31 \pm 0.14$ \\
\hline
\end{tabular}

${ }^{*} p<0.001$ versus SD rats (one-way ANOVA followed by Newman-Keuls' multiple comparison posttest).

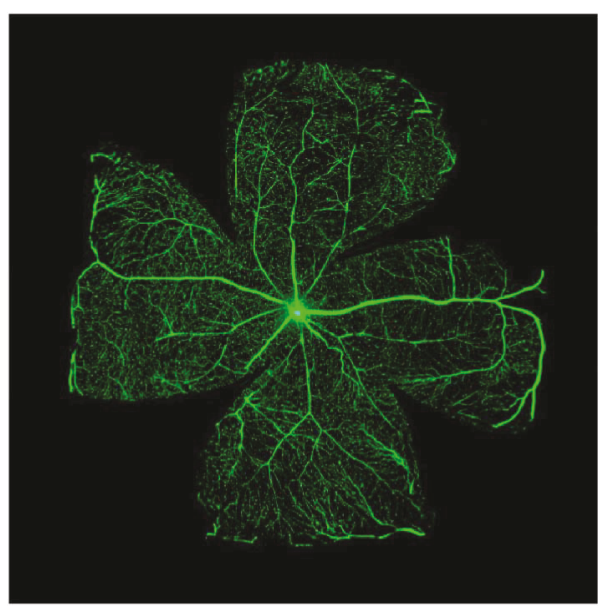

(a)

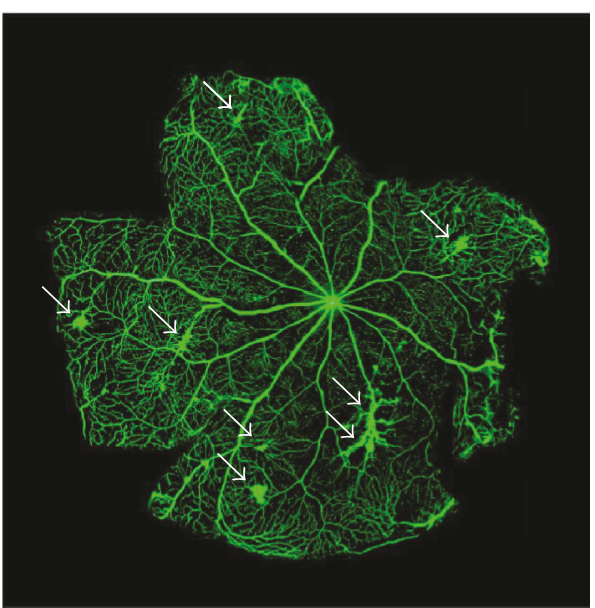

(b)

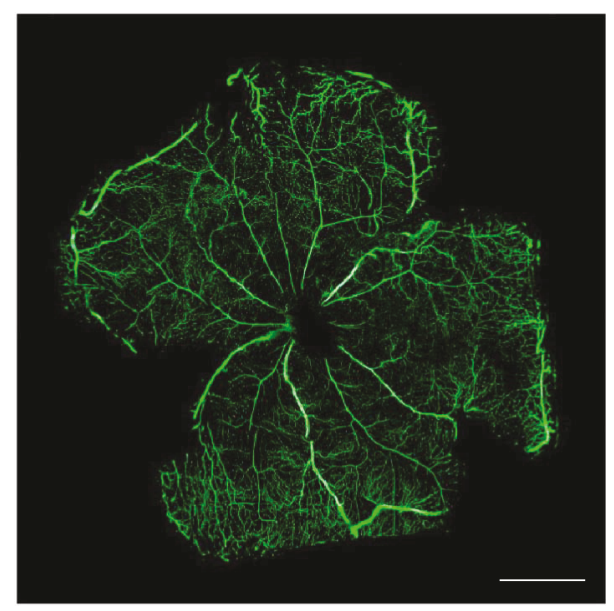

(c)

FIGURE 3: Effects of UPARANT on fluorescein leakage. $(\mathrm{a}-\mathrm{c})$ Fluorescein dextran microscopy performed soon after intraventricular injection of fluorescein solution in control SD (a) and SDT rats either vehicle-treated (b) or UPARANT-treated (c). Arrows in (b) point to hyperfluorescent areas in the retina of untreated SDT rats. In comparison with SD rats, untreated SDT rats showed abnormal retinal vasodilatation together with severe fluorescein leakage while the leakage is prevented by UPARANT administration. Scale bar: 1 mm. Fluorescein dextran microscopy was performed on 4 retinas (from untreated and vehicle-treated SDT rats) and 6 retinas (from SD control rats and UPARANT-treated SDT rats). The retinas originated from 2 or 3 different rats, respectively.

acts through mechanisms adjusting the expression level of the respective targets and/or a regulation of intracellular effectors induced by FPR activation was determined in both SDT rats and STZ rats, which were used for comparison. We first investigated whether high glucose affects the UPAR pathway and whether the preventive action of UPARANT depends on its effects on the uPAR/FPR system. As illustrated in Figures 9(a)-9(d), hyperglycemia enhanced retinal levels of uPAR/FPR transcripts in both SDT and STZ rats. In SDT rats, uPAR transcripts were increased by about 2.8-fold $(P<0.001)$, while FPR1, FPR2, and FPR3 transcripts were increased by about 3.9-fold, 4.3-fold, and 3.8-fold $(P<0.001)$, respectively. In STZ rats, uPAR and FPR transcripts were increased by about 5.2-fold $(P<0.001), 2.5$-fold $(P<0.01), 3.8$-fold $(P<0.001)$, and 4.1 -fold $(P<0.001)$, respectively. A similar increase was also found in vehicletreated SDT and STZ rats (not shown). In SDT rats, UPARANT reduced the upregulation of $\mathrm{UPAR}, \mathrm{FPR} 1$, and FPR2 


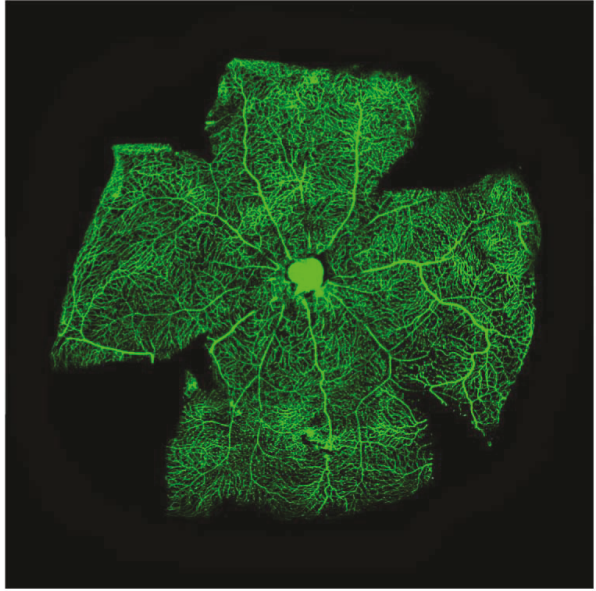

(a)

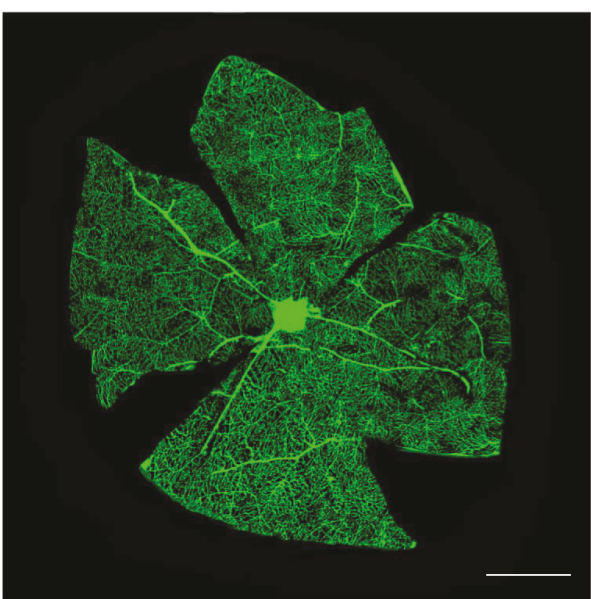

(b)

Figure 4: Retinal vascular phenotype. (a, b) Representive images of flat-mounted retinas from SD control rats (a) and untreated SDT rats (b) immunolabeled with a rat monoclonal antibody directed to CD31. The superficial vascular plexus is shown. No altered pattern of retinal vasculature in the superficial vascular plexus was observed in untreated SDT rats. CD31 immunohistochemistry was performed on 3 retinas from 3 different rats for each experimental condition. Scale bar: $1 \mathrm{~mm}$.

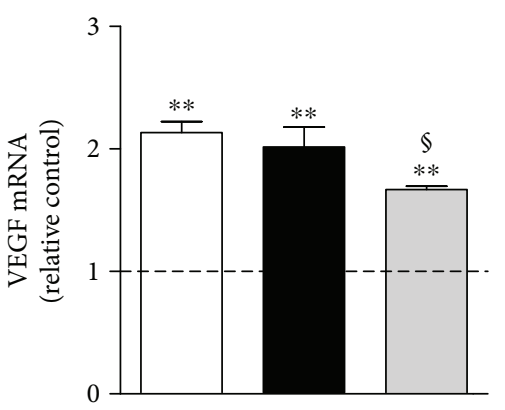

(a)

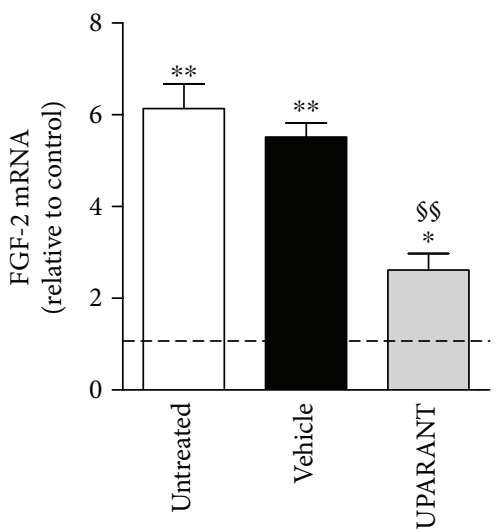

(c)

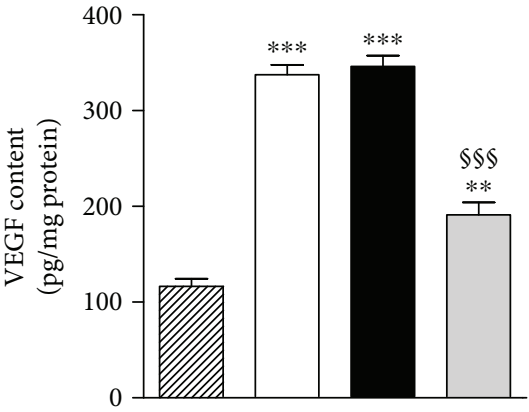

(b)

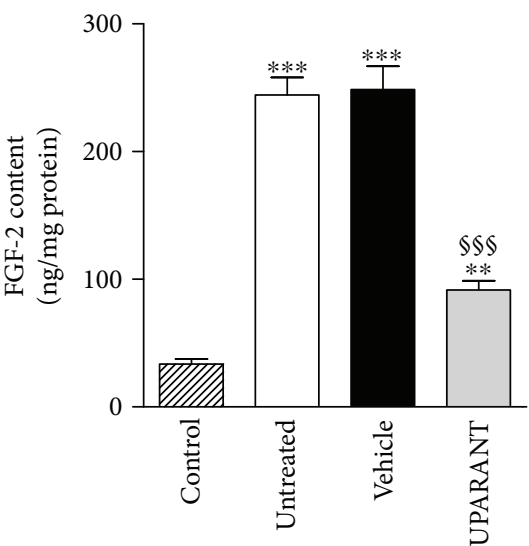

(d)

FIGURE 5: Effects of UPARANT on VEGF and FGF-2. (a, c) Transcript levels of VEGF (a) and FGF-2 (c) were evaluated by qPCR. Data were analyzed by the formula $2^{-\Delta \Delta C T}$ using Rpl13a and Hprt as internal standards. (b, d) Protein levels of VEGF (b) and FGF-2 (d) were evaluated by ELISA. In untreated or vehicle-treated SDT rats, levels of VEGF and FGF-2 were increased with respect to SD rats, while UPARANT treatment reduced this increase. ${ }^{*} P<0.05,{ }^{* *} P<0.01$, and ${ }^{* * *} P<0.001$ versus control; ${ }^{\S} P<0.05,{ }^{\$ \$} P<0.01$, and ${ }^{\circledR S \$} P<0.001$ versus vehicle (one-way ANOVA followed by Newman-Keuls' multiple comparison posttest; power values: 0.98 (a) and 0.99 (b-d)). Each column represents the mean \pm SEM of data from 3 independent samples, each containing 1 retina. 


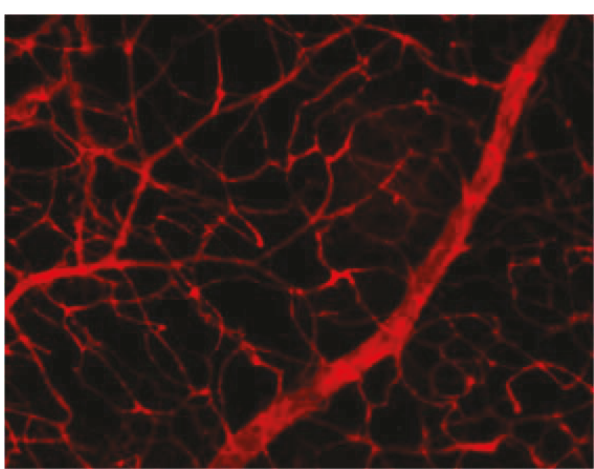

(a)

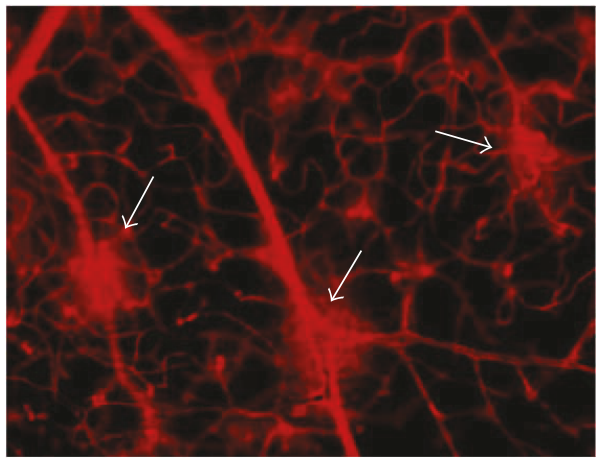

(c)

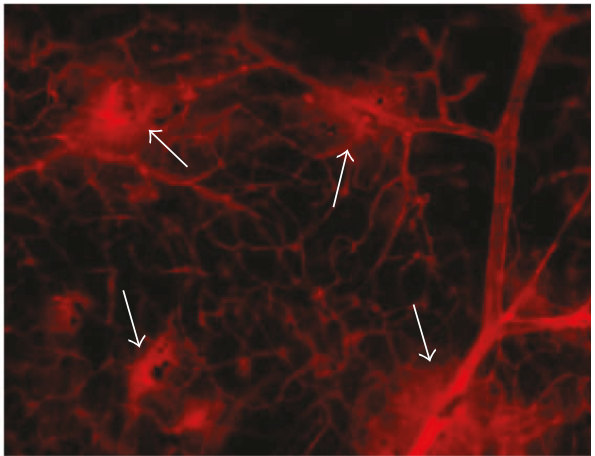

(b)

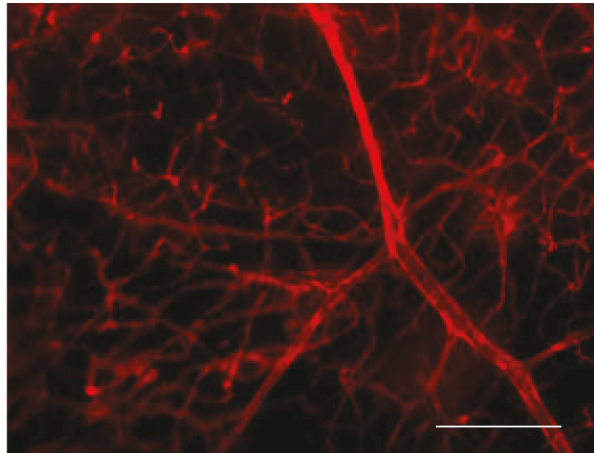

(d)

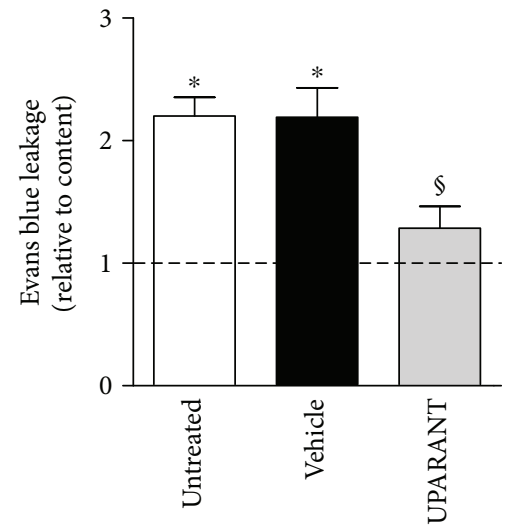

(e)

FIGURE 6: Effects of UPARANT on Evans blue leakage. (a-d) Blood-retinal vascular leakage as qualitatively evaluated with the Evans blue method in control SD (a) and SDT rats untreated (b), vehicle-treated (c), or UPARANT-treated (d). Arrows in (c) and (d) point to vascular leakage. Scale bar: $200 \mu \mathrm{m}$. (e) Diabetes-induced leakage as evaluated by the quantitative assessment of Evans blue dye extravasation. In untreated or vehicle-treated SDT rats, Evans blue dye leakage was increased with respect to SD rats, while UPARANT treatment prevented this increase. ${ }^{*} P<0.001$ versus control; ${ }^{\circledR} P<0.001$ versus vehicle (one-way ANOVA followed by Newman-Keuls' multiple comparison posttest; power value: 0.84 ). Each column represents the mean \pm SEM of data from 3 retinas from 3 different rats for each experimental condition. Three retinas from 3 different rats were used for each experimental condition.

by 1.7 -fold, 1.8-fold, and 1.9 -fold $(P<0.01)$ without affecting FPR3. In STZ rats, no effects of UPARANT on transcript levels of uPAR or FPRs could be observed. Transcript increase in both SDT and STZ rats was confirmed at the protein level. Representative blots from SD controls and untreated or UPARANT-treated SDT and STZ rats are depicted in Figure 10(a). The densitometric analysis (Figures 10(b)-10(e)) showed that uPAR, FPR1, FPR2, and FPR3 were increased by about 2.2-fold, 2.7- fold, 4.8-fold, and 4.9-fold $(P<0.001)$, respectively, in SDT rats, and by about 2.5-fold, 3.3-fold, 4.4-fold, and 4.1-fold $(P<0.001)$, respectively, in STZ rats. A similar increase was also found in vehicle-treated SDT and STZ rats (not shown).

The effects of UPARANT on factors that are downstream to FPRs [16] and are known to mediate the transcription of inflammatory factors, including cytokines [17], were also investigated in both SDT and STZ rats. 


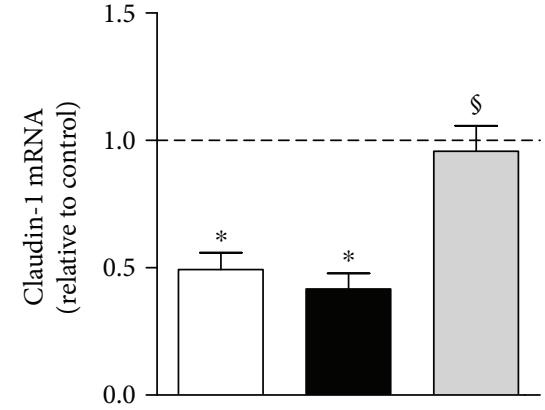

(a)

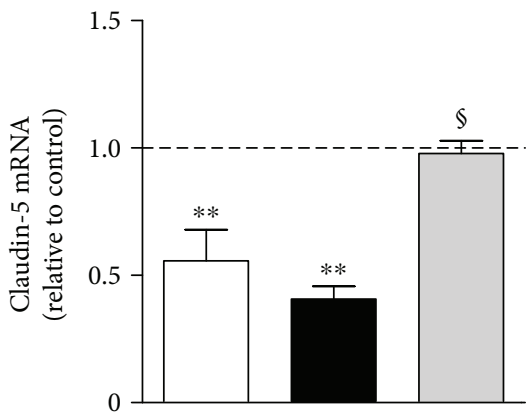

(c)

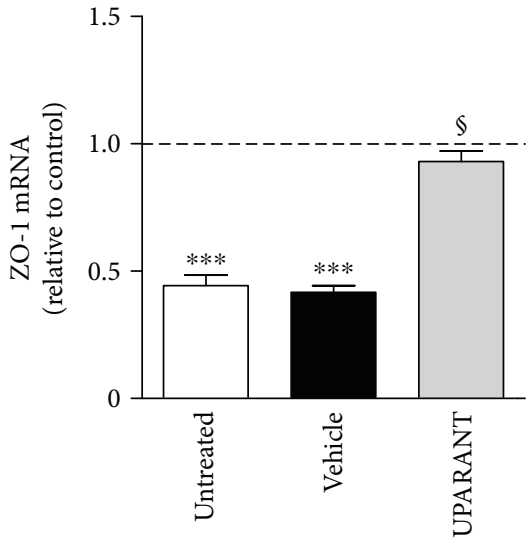

(e)

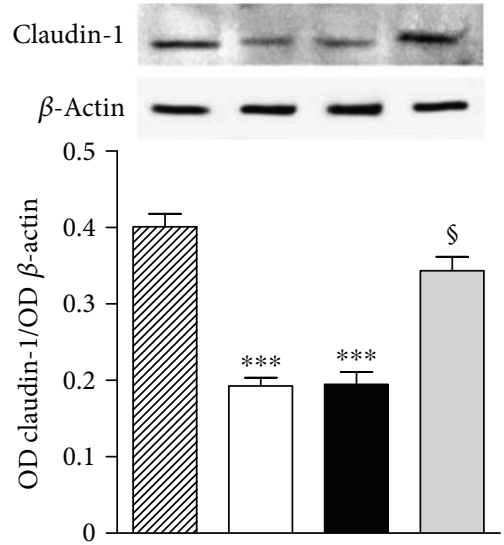

(b)

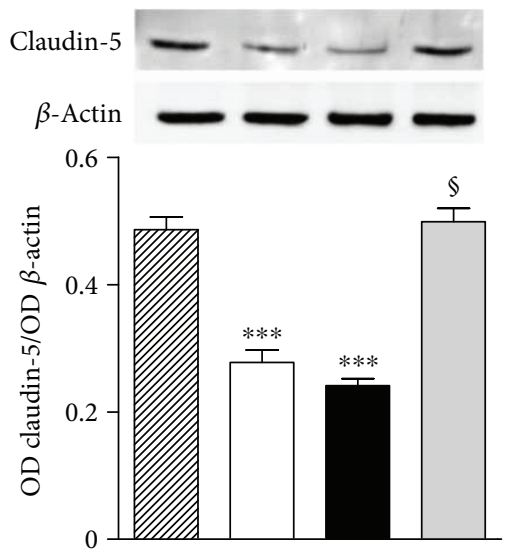

(d)

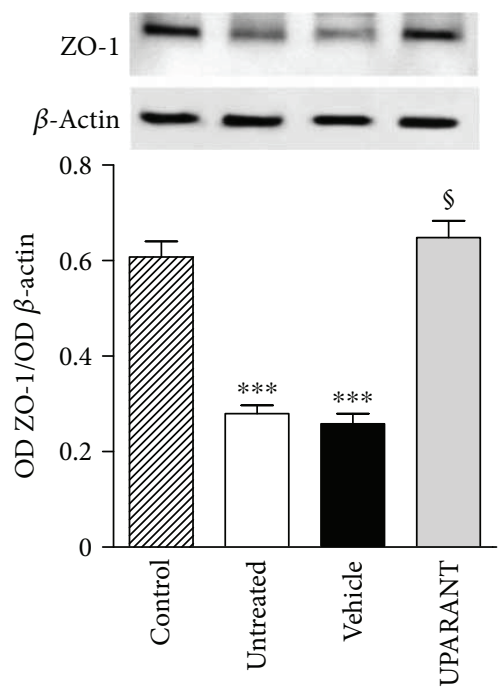

(f)

Figure 7: Effects of UPARANT on levels of BRB markers. (a, c, e) Transcript levels of the BRB markers claudin-1 (a), claudin-5 (c), and ZO-1 (e) were evaluated by qPCR. Data were analyzed by the formula $2^{-\Delta \Delta C T}$ using Rpl13a and Hprt as internal standards. (b, d, f) Protein levels of claudin-1 (b), claudin-5 (d), and ZO-1 (f) were evaluated by Western blot and densitometric analysis using $\beta$-actin as the loading control. In untreated or vehicle-treated SDT rats, levels of BRB markers were decreased with respect to SD rats, while UPARANT treatment prevented this decrease. ${ }^{*} P<0.05,{ }^{* *} P<0.01$, and ${ }^{* * *} P<0.001$ versus control; ${ }^{\circledR} P<0.001$ versus vehicle (one-way ANOVA followed by NewmanKeuls' multiple comparison posttest; power values: 0.85 (a), 0.99 (b), 0.85 (c), and 0.99 (d-f)). Each column represents the mean \pm SEM of data from 3 independent samples, each containing 1 retina. 


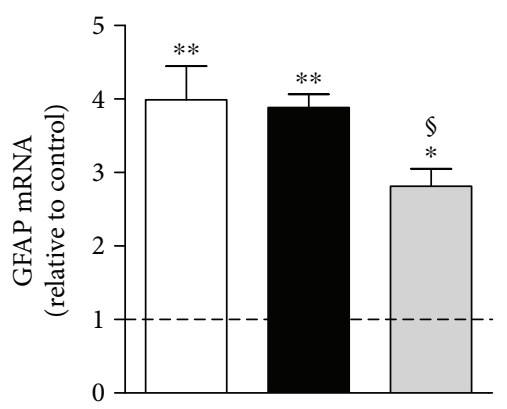

(a)

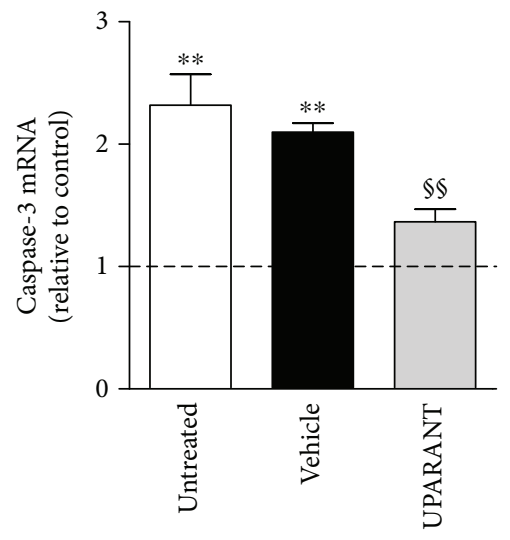

(c)
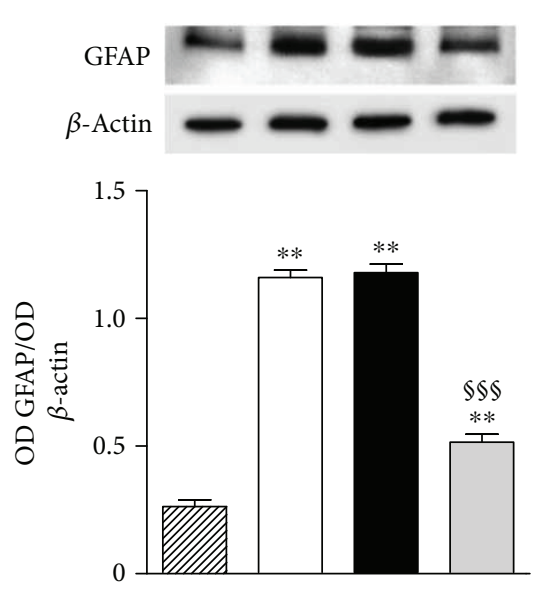

(b)
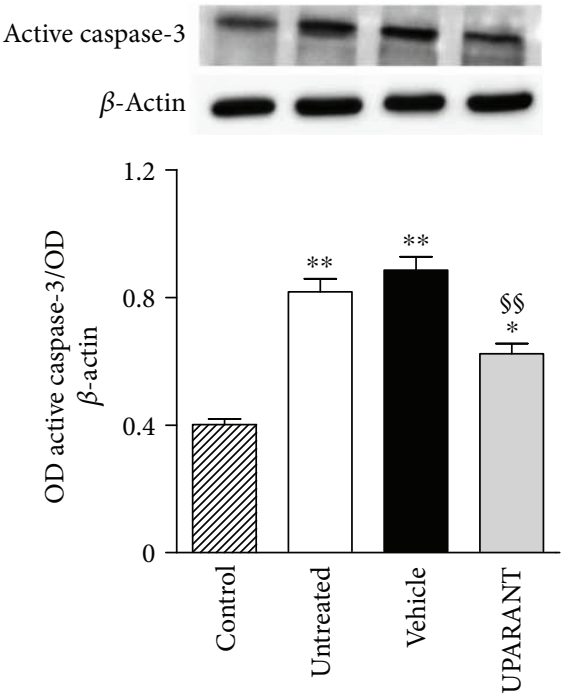

(d)

FIGURE 8: Effects of UPARANT on gliosis and retinal cell death. (a, c) Transcript levels of GFAP (a) and caspase-3 (c) were evaluated by qPCR. Data were analyzed by the formula $2^{-\Delta \Delta C T}$ using Rpl13a and Hprt as internal standards. (b, d) Protein levels of GFAP (b) and active caspase-3 (d) were evaluated by Western blot and densitometric analysis using $\beta$-actin as the loading control. In untreated or vehicle-treated SDT rats, levels of GFAP and caspase- 3 were increased with respect to SD rats, while UPARANT treatment reduced this increase. ${ }^{*} P<0.01$ and ${ }^{* *} P<0.001$ versus control; ${ }^{\circledR} P<0.05,{ }^{\circledR S} P<0.01$, and ${ }^{\circledR \$ \$} P<0.001$ versus vehicle (one-way ANOVA followed by Newman-Keuls' multiple comparison posttest; power values: 0.94 (a), 0.99 (b), 0.87 (c), and 0.99 (d)). Each column represents the mean \pm SEM of data from 3 independent samples, each containing 1 retina.

As shown in Figures 11(a)-11(c), in both models, high glucose increased the phosphorylation of STAT3 at $\mathrm{Tyr}^{705}$ and NF- $\kappa$ B p65 at Ser ${ }^{276}$ by about 5.6-fold and 2.9-fold $(P<0.001)$, respectively, in SDT rats, and by about 10.3 -fold and 3.3-fold $(P<0.001)$, respectively, in STZ rats. In both SDT and STZ rats, the increase in transcription factor phosphorylation was significantly reduced by UPARANT by 3.4fold and 1.6-fold in SDT $(P<0.001)$ and by 3.0 -fold and 1.7 fold in STZ rats $(P<0.01)$. We also found that untreated SDT rats were characterized by transcript levels of TNF$\alpha$, IL- $1 \beta$, and IL- 6 by 4.0 -fold $(P<0.001), 2.3$-fold $(P<$ $0.001)$, and 2.4 -fold $(P<0.01)$ higher than in SD controls (Figures 12(a), 12(c), and 12(e)). A similar increase was also observed in vehicle-treated SDT rats. UPARANT reduced the transcript upregulation by 1.4 -fold $(P<0.01)$, 1.7-fold
$(P<0.01)$, and 1.3 -fold $(P<0.05)$, respectively. Comparable effects of persisting hyperglicemia on inflammatory factors were also determined at the protein level with TNF- $\alpha$, IL$1 \beta$, and IL- 6 in untreated or vehicle-treated SDT rats roughly 5.0-fold, 6.0-fold, and 7.9-fold higher $(P<0.001)$ than in SD controls (Figures 12(b), 12(d), and 12(f)). UPARANT reduced protein upregulation by 1.6 -fold $(P<0.01), 2.1$-fold $(P<0.001)$, and 1.6-fold $(P<0.01)$, respectively.

\section{Discussion}

The present study demonstrates for the first time the preventive efficacy of UPARANT on retinal damage that characterizes the SDT rat as a model approximating type 2 diabetes. Presently, hyperglycemia in type 2 diabetic 


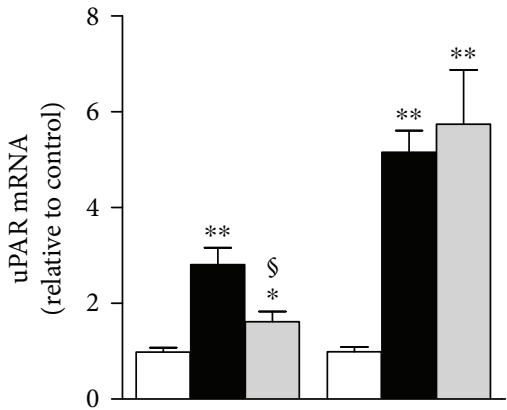

(a)

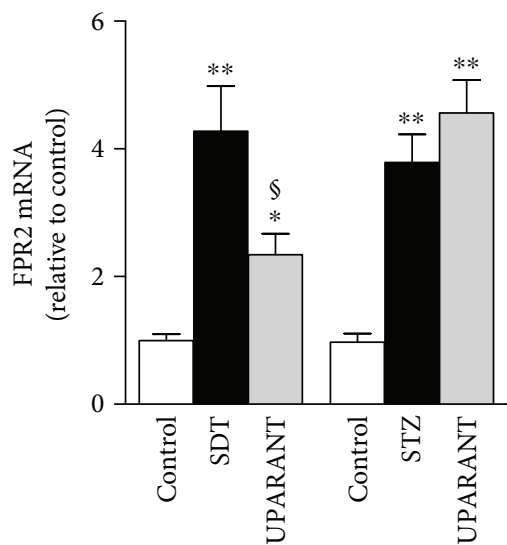

(c)

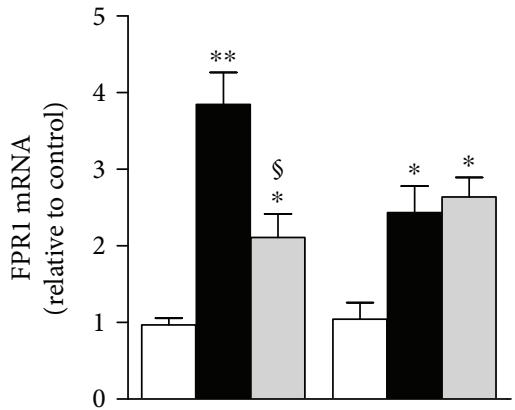

(b)

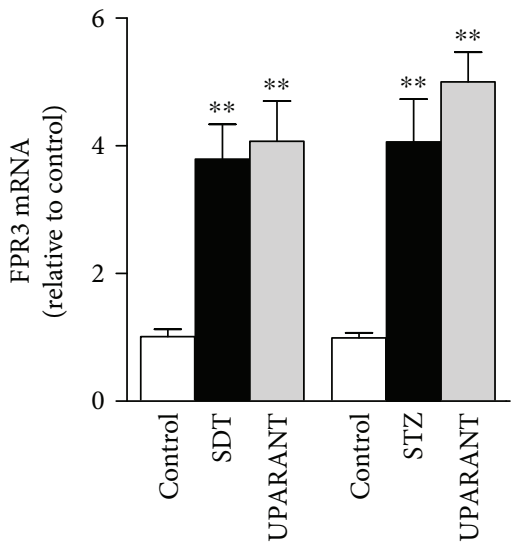

(d)

FIGURE 9: Effects of UPARANT on upregulated levels of uPAR and FPRs. (a-d) Transcript levels of uPAR (a), FPR1 (b), FPR2 (c), and FPR3 (d) were evaluated by qPCR in control SD and in SDT or STZ rats either untreated or treated with UPARANT. Data were analyzed by the formula $2^{-\Delta \Delta C T}$ using Rpl13a and Hprt as internal standards. Hyperglycemia enhanced retinal levels of uPAR/FPR transcripts in both SDT and STZ rats. In SDT rats, UPARANT reduced the upregulation of uPAR, FPR1, and FPR2 without affecting FPR3. In STZ rats, no effects of UPARANT on transcript levels of uPAR or FPRs could be observed. ${ }^{*} P<0.01$ and ${ }^{* *} P<0.001$ versus control SD rats; ${ }^{\circledR} P<0.01$ versus untreated SDT rats (one-way ANOVA followed by Newman-Keuls' multiple comparison posttest; power values: 0.86 (a), 0.93 (b), 0.91 (c), and $0.96(\mathrm{~d}))$. Each column represents the mean \pm SEM of data from 3 independent samples, each containing 1 retina.

patients is controlled by oral hypoglycemic and antihyperglycemic drugs although they are not always successful in preventing the onset of DR requiring anti-VEGF treatments that are given when the disease has become vision threatening. A distinctive characteristic of UPARANT is that it prevents DR likely through a dual action, (i) by directly regulating its target expression and (ii) by acting at UPAR crosstalk with FPRs, influencing the activity of transcription factors regulating the expression of inflammatory markers. UPARANT preventive effects demonstrated here also support the effectiveness of systemic administration although its safety in SDT rats still remains to be evaluated.

\subsection{Characterization of the SDT Model and UPARANT} Efficacy. Although no rodent models of type 2 diabetes show the same features of human DR, SDT rats display retinal lesions that closely resemble the human disease including vascular abnormalities leading to reduced barrier properties. Indeed, fluorescein leakage and Evans blue extravasation found here are both indicative of BRB breakdown as also confirmed by altered levels of tight junction components of retinal endothelial cells including claudin-
1, claudin-5, and ZO-1 that play an important role in $\mathrm{BRB}$ integrity [18]. In particular, they are downregulated in the diabetic retina $[19,20]$ and in retinal endothelial cells cultured in a high glucose [21, 22]. The additional finding that retinal levels of VEGF and FGF-2 in SDT rats are higher than in SD controls is in line with previous results demonstrating VEGF upregulation in the retina of SDT rats $[23,24]$. On the other hand, the lack of alterations in the pattern of retinal vasculature found here is in line with the very low incidence of retinal neovascular formation as described at about 20 weeks after diabetes onset in SDT rats [24]. This is in line with the fact that VEGF not only stimulates vessel proliferation but also plays a role as vasopermeability factor in the diabetic retina [25]. In this respect, Müller cells and activated microglia secrete vasoactive and inflammatory molecules including VEGF [26]. In particular, Müller cells are stimulated by FGF-2 to produce VEGF [27] that, in turn, participates in BRB breakdown by reducing the level of tight junction proteins [28].

Vascular abnormalities leading to reduced barrier properties may be reflected in depressed ERGs, which are established at relatively early stages of the disease [5]. In fact, 


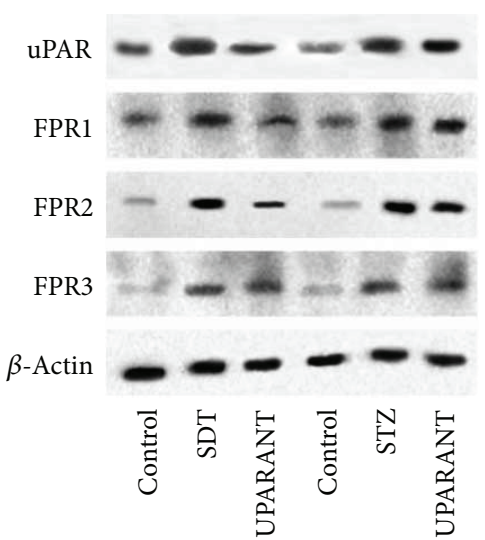

(a)

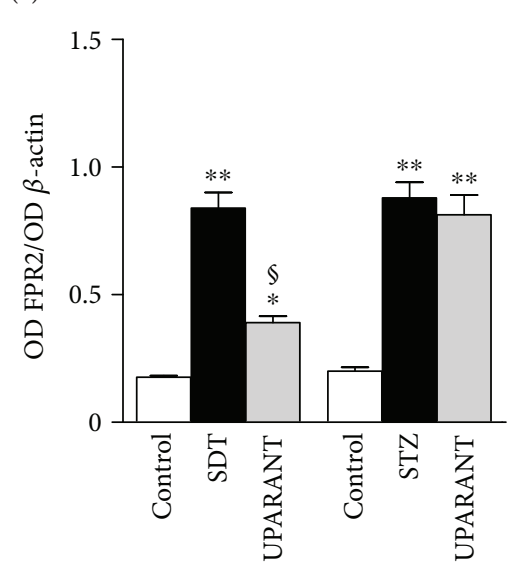

(d)

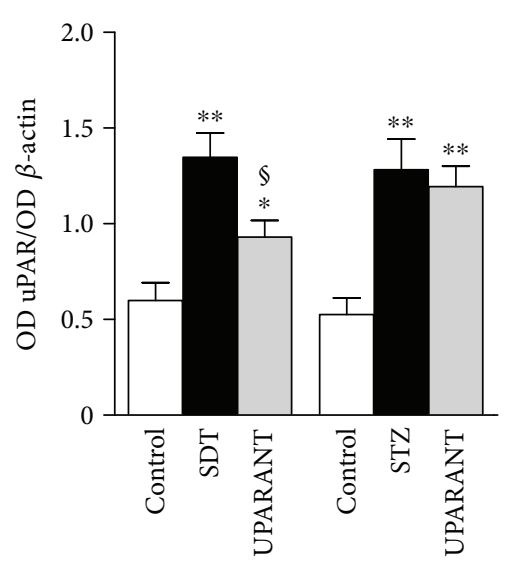

(b)

(c)

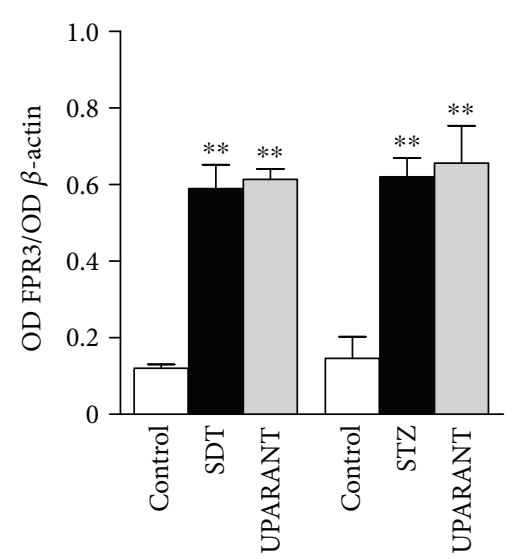

(e)

FIgURE 10: Effects of UPARANT on upregulated levels of uPAR and FPRs. (a) Representative blots from SD controls and untreated or UPARANT-treated SDT and STZ rats. (b-e) Protein levels of uPAR (b), FPR1 (c), FPR2 (d), and FPR3 (e) were evaluated by the densitometric analysis of the blots depicted in (a) using $\beta$-actin as the loading control. Hyperglycemia enhanced retinal levels of uPAR/FPR proteins in both SDT and STZ rats. In SDT rats, UPARANT reduced the upregulation of uPAR, FPR1, and FPR2 without affecting FPR3. In STZ rats, no effects of UPARANT on protein levels of uPAR or FPRs could be observed. ${ }^{*} P<0.01$ and ${ }^{* *} P<0.001$ versus control SD rats; ${ }^{\$} P<0.01$ versus untreated SDT rats (one-way ANOVA followed by Newman-Keuls' multiple comparison posttest; power values: 0.87 (b), 0.99 (c, d), and 0.98 (e)). Each column represents the mean \pm SEM of data from 3 independent samples, each containing 1 retina.

about 20 weeks of hyperglycemia lead to a $50 \%$ reduction in both the a-wave and the b-wave suggesting an involvement of both the outer and the inner retina. On the other hand, hyperglicemia-induced ERG dysfunction is reported even before $\mathrm{BRB}$ breakdown indicating that neurodegenerative processes are occurring before the capillary alterations [29]. In this line, additional retinal damages found here including gliosis and retinal cell death are in agreement with previous findings in SDT rats in which GFAP accumulation and caspase-3 activation have been demonstrated in inner and outer retinal layers $[30,31]$. In this respect, in the diabetic retina, neurodegenerative processes affect mainly ganglion cells and amacrine cells although photoreceptor death and alterations in the expression of phototrasduction proteins have been also reported [29].

UPARANT dose used here is in line with that used in the STZ model by Cammalleri et al. [12] who also demonstrated that UPARANT reaches the retinal target when subcutaneously administered without any histopathologic alteration of the liver and kidney, the most important organs for detoxification processes, thus indicating the subcutaneous delivery as a promising route to enter the posterior chamber of the eye in the STZ model.

UPARANT-induced prevention of BRB loss, Müller cell gliosis, and retinal cell death shown here in SDT rats is likely to contribute to maintaining retinal integrity as also demonstrated by preventive efficacy of the drug on ERG dysfunction. Preventive action of UPARANT on BRB breakdown has been previously demonstrated in mouse models of neovascular ocular diseases $[10,11]$. In addition, UPARANT prevents the VEGF-induced permeability in a monolayer of human retinal endothelial cells [32] and efficiently treats BRB leakage in the STZ model in which VEGF and FGF-2 upregulation is also prevented [12]. In this respect, drugs that reduce retinal levels of VEGF are found to prevent ERG dysfunction or reduce BRB leakage thus limiting DR progression in SDT rats [33-35]. In addition, ranirestat, an inhibitor of the enzyme aldose reductase, which has an 


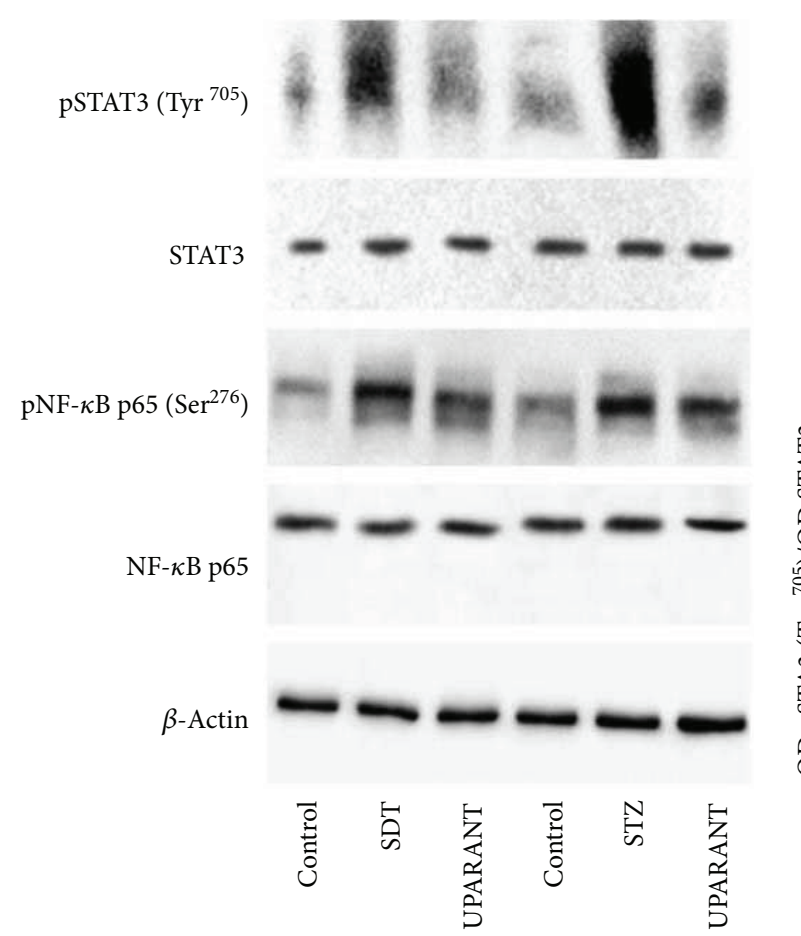

(a)

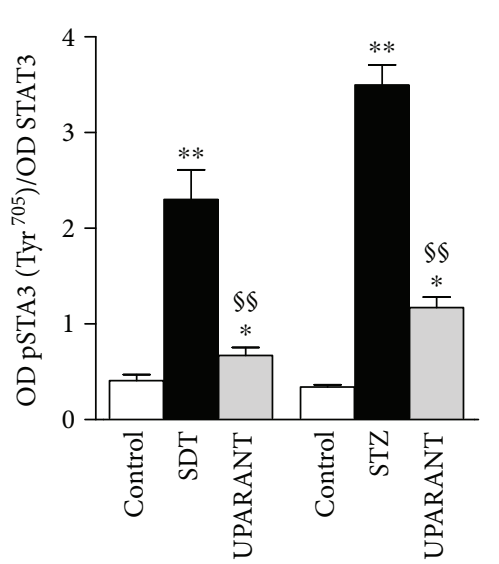

(b)

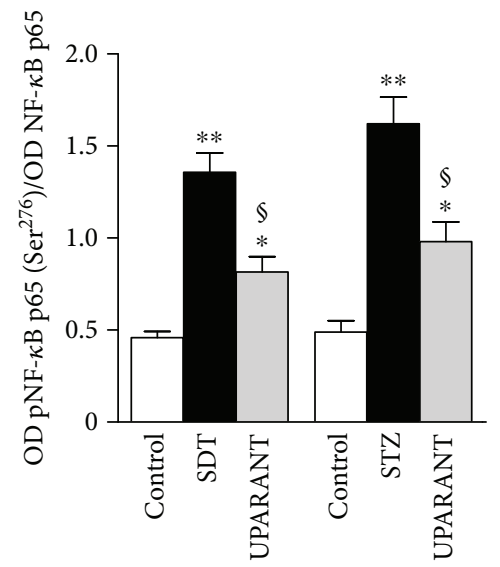

(c)

FIGURE 11: Effects of UPARANT on upregulated levels of transcription factors. (a) Representative blots from SD controls and untreated or UPARANT-treated SDT and STZ rats. (b, c) Protein levels of pSTAT3 $\left(\mathrm{Tyr}^{205}\right.$ ) (b) and pNF- $\kappa \mathrm{B}$ p65 (Ser ${ }^{276}$ ) (c) were evaluated by the densitometric analysis of the blots depicted in (a) using STAT3 or NF- $\kappa$ B p65 as the loading controls. Hyperglycemia enhanced the phosphorylation of STAT3 at $\mathrm{Tyr}^{705}$ and NF- $\kappa \mathrm{B}$ p65 at $\mathrm{Ser}^{276}$ in both SDT and STZ rats. The increase in transcription factor phosphorylation was significantly reduced by UPARANT in both SDT and STZ rats. ${ }^{*} P<0.01$ and ${ }^{* *} P<0.001$ versus control SD rats; ${ }^{\S} P<0.01$ and ${ }^{\$} P<0.001$ versus untreated SDT or STZ rats (one-way ANOVA followed by Newman-Keuls' multiple comparison posttest; power values: $0.99(\mathrm{~b}, \mathrm{c})$ ). Each column represents the mean \pm SEM of data from 3 independent samples, each containing 1 retina.

early role in the development of DR [36], exerts neuroprotective effects by reducing GFAP accumulation and preventing hyperglycemia-associated structural damage of the retina [31].

4.2. Mechanisms Underlying UPARANT Effects. As shown by the present results, the SDT model is characterized by an increased expression of uPAR and FPRs at the transcript and the protein level thus confirming a direct link between their upregulation and DR development. Of the FPRs, FPR2 plays a proinflammatory role as demonstrated in human carotid atherosclerotic lesions, whereas a lower inflammatory response has been observed in macrophages with FPR2 deletion [37]. The additional fact that UPARANT reduces upregulated levels of uPAR, FPR1, and FPR2, without any effect on FPR3, is in line with the finding that the drug has been designed to mimic the sequence through which uPAR interacts with FPR1 and FPR2, but not with FPR3 [9].

Many drug targets thought to be suitable for therapeutic purposes are subjected to positive or negative feedback loops upon chemical perturbations, which might even account for the development of drug tolerance [38]. In particular, members of the G-protein-coupled receptor family when serving as known targets, are regulated upon drug treatment by several mechanisms including receptor desensitization, endocytosis, or regulation of the cellular receptor content $[39,40]$. Here, we found that UPARANT treatment in two different rat models of DR, the SDT rat and the STZ rat, achieves the same efficacy, either preventive or curative, through partially different responses. In fact, in the SDT model, UPARANT triggers a negative feedback loop that downregulates the levels of its targets thus presumably rendering the treatment more effective than if it would act at the receptor downstream level only. In the STZ model, in contrast, the uPAR pathway is not influenced by UPARANT suggesting that drug efficacy is solely dependent on potentially switching off the intracellular pathway downstream FPRs. Similarly, in a mouse model of wet AMD, UPARANT has been shown to mitigate laser-induced choroidal neovascularization by inhibiting FPR-mediated regulation of transcription factors coupled to angiogenesis and inflammation without affecting the expression levels of the uPAR/ FPR pathway [10].

There are several examples of long-term effects of drugs that are mainly due to the modulation of drug target expression presumably because of the chronic treatment in respect to acute administration. For instance, in rodent models of stroke, the antidiabetic drug metformin can reduce the ischemic events by direcly influencing its molecular targets when administered chronically, but not when administered acutely [41]. In the SDT model, DR lasts several months thus 


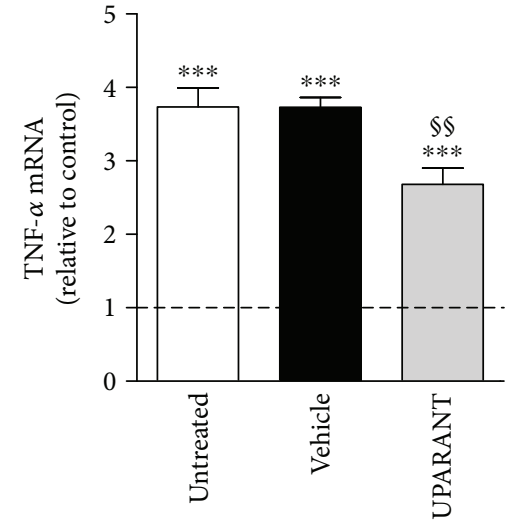

(a)

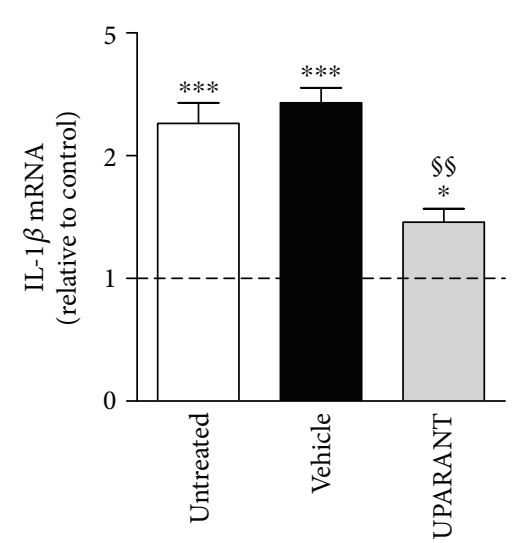

(c)

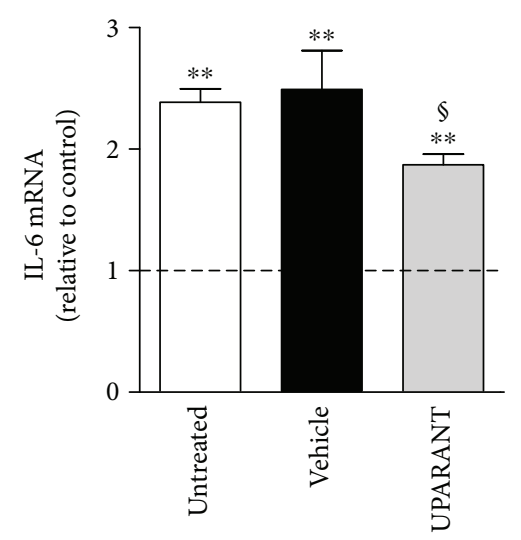

(e)

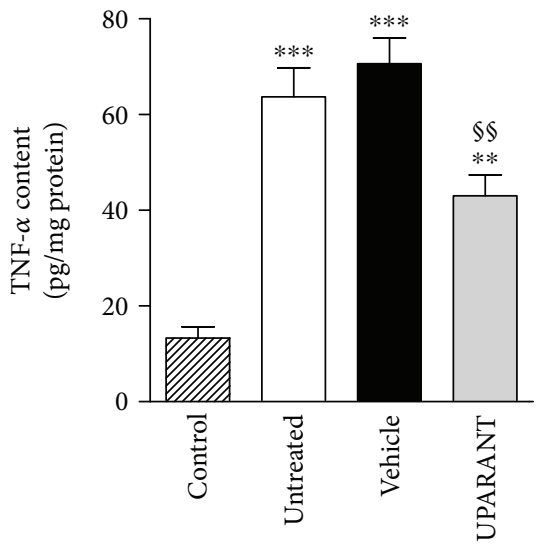

(b)

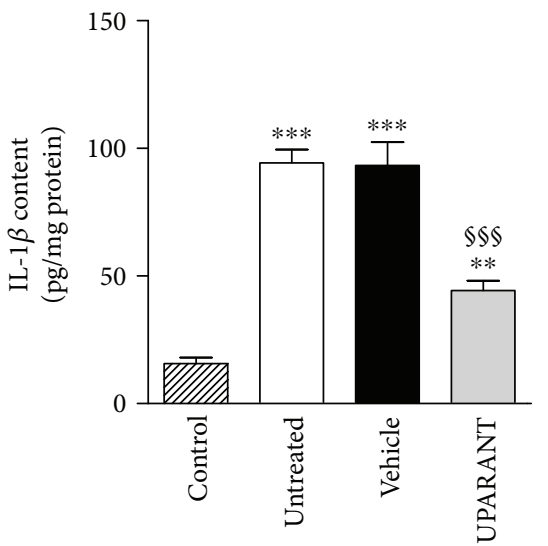

(d)

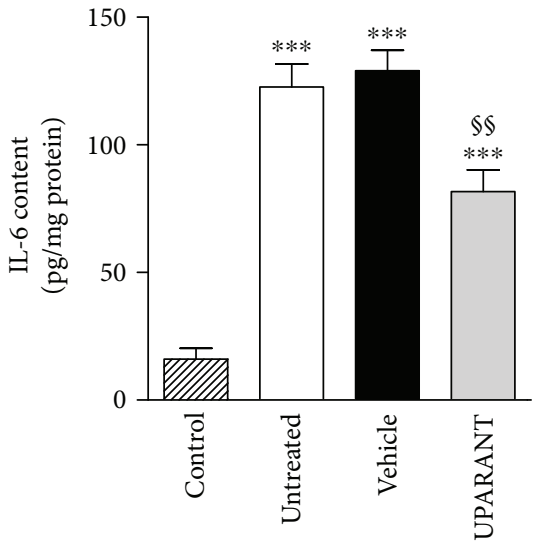

(f)

Figure 12: Effects of UPARANT on inflammatory markers. (a, c, e) Transcript levels of TNF- $\alpha$ (a), IL-1 $\beta$ (c), and GFAP (e) were evaluated by qPCR. Data were analyzed by the formula $2^{-\Delta \Delta C T}$ using Rpl13a and Hprt as internal standards. (b, d, f) Protein levels of TNF- $\alpha$ (b), IL-1 $\beta$ (d), and GFAP (f) were evaluated by ELISA. In untreated or vehicle-treated SDT rats, levels of inflammatory markers were increased with respect to SD rats, while UPARANT treatment reduced this increase. ${ }^{*} P<0.05,{ }^{* *} P<0.01$, and ${ }^{* * *} P<0.001$ versus control SD rats; ${ }^{\$} P<0.05,{ }^{\circledR} P<0.01$, and ${ }^{\circledR \$ S} P<0.001$ versus vehicle (one-way ANOVA followed by Newman-Keuls' multiple comparison posttest; power values: $0.99(\mathrm{a}-\mathrm{d}), 0.85$ (e), and 0.99 (f)). Each column represents the mean \pm SEM of data from 3 independent samples, each containing 1 retina.

requiring long-lasting treatment, whereas, in the STZ model, UPARANT efficiently counteracts DR signs after 5 days of administration [12]. In both models, UPARANT inhibits FPR-mediated regulation of transcription factors coupled to inflammation. In SDT rats, consequently, preventive administration of UPARANT reduces upregulated levels of inflammatory markers in response to high glucose in line with what found in the STZ model, in which UPARANT decreases inflammation when administered in a therapeutic regimen [12]. Inflammatory factors determined here 
include inflammatory cytokines such as TNF- $\alpha$, IL- $1 \beta$, and IL6. Among them, TNF- $\alpha$ regulates the expression of IL- $1 \beta$ and IL-6, while IL- $1 \beta$ activates IL- 6 production [42]. Inflammatory molecules may induce Müller cell gliosis, as evidenced by increased GFAP expression, and gliotic Müller cells express a wide variety of inflammatory factors, including cytokines [43]. In addition, UPARANT anti-inflammatory activity participates to the inhibition of the angiogenic phenotype by endothelial cells in response to the vitreous fluid from patients with proliferative DR, which is characterized by high levels of angiogenic and inflammatory factors [44]. Moreover, UPARANT action as an anti-inflammatory drug has been recently demonstrated in animal models of inflammation [45]. In this respect, as therapies targeting VEGF do not intervene on inflammatory processes, UPARANT benefits in DR may be greater than those of most anti-VEGF therapies. Consistently, a main role of anti-inflammatory drugs in slowing down the progression of DR lesions has been recently recognized [46].

\section{Conclusions}

The extrapolation of these experimental findings to the clinic is not straightforward although the SDT model approximates at least in part type 2 diabetes. However, the present study provides evidence that preventive administration of UPARANT and continuing regularly along diabetes progression counteracts DR development thus presumably protecting the retina from further worsening of the pathology. In addition, UPARANT preventive effect demonstrated here supports the effectiveness of the systemic route although drug safety still remains to be evaluated. Therefore, the possibility to use a systemic drug that, by slowing down DR progression, may delay the use of intraocularly delivered anti-VEGF agents may be viewed as an added value of UPARANT.

\section{Disclosure}

Sooft Fidia Pharma had no role in the design of the study; in the collection, analyses, or interpretation of data; in the writing of the manuscript; and in the decision to publish the results.

\section{Conflicts of Interest}

Maurizio Cammalleri received a research grant from Sooft Italia S.p.A. Dario Rusciano is an employee of Sooft Fidia Pharma. Mario De Rosa and Vincenzo Pavone are the holders of UPARANT patent. Massimo Dal Monte, Filippo Locri, Stefania Marsili, Liliana Lista, and Paola Bagnoli declare no conflicts of interest.

\section{Authors' Contributions}

Dario Rusciano and Paola Bagnoli conceived and designed the study. Maurizio Cammalleri, Massimo Dal Monte, and Filippo Locri performed the experiments. Mario De Rosa and Vincenzo Pavone designed and characterized the drug.
Liliana Lista synthesized the drug. Maurizio Cammalleri, Massimo Dal Monte, Filippo Locri, Stefania Marsili, Mario De Rosa, Vincenzo Pavone, Dario Rusciano, and Paola Bagnoli analyzed the data and interpreted the results. Paola Bagnoli and Massimo Dal Monte wrote the paper, and Maurizio Cammalleri participated to its writing. All authors contributed to the proofreading and correction of the manuscript. All authors have approved the final article. Maurizio Cammalleri and Massimo Dal Monte equally contributed to the study.

\section{Acknowledgments}

The authors would like to thank Gino Bertolini for technical assistance. This work was supported by grants from Italian Ministero della Salute (RF-201102351158; Paola Bagnoli; Roma, Italy), Sooft Italia S.p.A (Maurizio Cammalleri; Montegiorgio, Italy), and European Union-Fondi Europei per lo Sviluppo Regionale, Italian Ministero dell'Istruzione e dell'Università, and Italian Ministero dello Sviluppo Economico (PON01 02464; Mario De Rosa and Vincenzo Pavone; Roma, Italy).

\section{References}

[1] D. Deshpande, M. Harris-Hayes, and M. Schootman, "Epidemiology of diabetes and diabetes-related complications," Physical Therapy, vol. 88, no. 11, pp. 1254-1264, 2008.

[2] R. Robinson, V. A. Barathi, S. S. Chaurasia, T. Y. Wong, and T. S. Kern, "Update on animal models of diabetic retinopathy: from molecular approaches to mice and higher mammals," Disease Models \& Mechanisms, vol. 5, no. 4, pp. 444-456, 2012.

[3] A. K. Lai and A. C. Lo, "Animal models of diabetic retinopathy: summary and comparison," Journal of Diabetes Research, vol. 2013, Article ID 106594, 29 pages, 2013.

[4] M. Shinohara, T. Masuyama, T. Shoda et al., "A new spontaneously diabetic non-obese Torii rat strain with severe ocular complications," International Journal of Experimental Diabetes Research, vol. 1, no. 2, pp. 89-100, 2000.

[5] T. Sasase, "Pathophysiological characteristics of diabetic ocular complications in spontaneously diabetic torii rat," Journal of Ophthalmology, vol. 2010, Article ID 615641, 7 pages, 2010.

[6] A. B. El-Remessy, T. Franklin, N. Ghaley et al., "Diabetesinduced superoxide anion and breakdown of the bloodretinal barrier: role of the VEGF/UPAR pathway," PLoS One, vol. 8, no. 8, article e71868, 2013.

[7] A. B. El-Remessy, M. A. Behzadian, G. Abou-Mohamed, T. Franklin, R. W. Caldwell, and R. B. Caldwell, "Experimental diabetes causes breakdown of the blood-retina barrier by a mechanism involving tyrosine nitration and increases in expression of vascular endothelial growth factor and urokinase plasminogen activator receptor," The American Journal of Pathology, vol. 162, no. 6, pp. 1995-2004, 2003.

[8] D. Navaratna, G. Menicucci, J. Maestas, R. Srinivasan, P. McGuire, and A. Das, "A peptide inhibitor of the urokinase/urokinase receptor system inhibits alteration of the blood-retinal barrier in diabetes," The FASEB Journal, vol. 22, no. 9, pp. 3310-3317, 2008. 
[9] M. V. Carriero, K. Bifulco, M. Minopoli et al., "UPARANT: a urokinase receptor-derived peptide inhibitor of VEGF-driven angiogenesis with enhanced stability and in vitro and in vivo potency," Molecular Cancer Therapeutics, vol. 13, no. 5, pp. 1092-1104, 2014.

[10] M. Cammalleri, M. Dal Monte, F. Locri et al., "The urokinase receptor-derived peptide UPARANT mitigates angiogenesis in a mouse model of laser-induced choroidal neovascularization," Investigative Ophthalmology \& Visual Science, vol. 57, no. 6, pp. 2600-2597, 2016.

[11] M. Dal Monte, S. Rezzola, M. Cammalleri et al., "Antiangiogenic effectiveness of the urokinase receptor-derived peptide UPARANT in a model of oxygen-induced retinopathy," Investigative Ophthalmology \& Visual Science, vol. 56, no. 4, pp. 2392-2407, 2015.

[12] M. Cammalleri, F. Locri, S. Marsili et al., "The urokinase receptor-derived peptide UPARANT recovers dysfunctional electroretinogram and blood-retinal barrier leakage in a rat model of diabetes," Investigative Ophthalmology \& Visual Science, vol. 58, no. 7, pp. 3138-3148, 2017.

[13] K. I. Naka and W. A. Rushton, "S-potentials from colour units in the retina of fish (Cyprinidae)," The Journal of Physiology, vol. 185, no. 3, pp. 536-555, 1966.

[14] T. Okuno, H. Oku, T. Sugiyama, and T. Ikeda, "Electroretinographic study of spontaneously diabetic Torii rats," Documenta Ophthalmologica, vol. 117, no. 3, pp. 191-196, 2008.

[15] D. J. Feenstra, E. C. Yego, and S. Mohr, "Modes of retinal cell death in diabetic retinopathy," Journal of Clinical \& Experimental Ophthalmology, vol. 04, no. 5, article 298, 2013.

[16] F. Cattaneo, G. Guerra, and R. Ammendola, "Expression and signaling of formyl-peptide receptors in the brain," Neurochemical Research, vol. 35, no. 12, pp. 2018-2026, 2010.

[17] Y. Fan, R. Mao, and J. Yang, "NF- $\kappa$ B and STAT3 signaling pathways collaboratively link inflammation to cancer," Protein \& Cell, vol. 4, no. 3, pp. 176-185, 2013.

[18] C. Kaur, W. S. Foulds, and E. A. Ling, "Blood-retinal barrier in hypoxic ischaemic conditions: basic concepts, clinical features and management," Progress in Retinal and Eye Research, vol. 27, no. 6, pp. 622-647, 2008.

[19] E. C. Leal, J. Martins, P. Voabil et al., "Calcium dobesilate inhibits the alterations in tight junction proteins and leukocyte adhesion to retinal endothelial cells induced by diabetes," Diabetes, vol. 59, no. 10, pp. 2637-2645, 2010.

[20] L. Fan and H. Yan, "FTY720 attenuates retinal inflammation and protects blood-retinal barrier in diabetic rats," Investigative Ophthalmology \& Visual Science, vol. 57, no. 3, pp. 1254-1263, 2016.

[21] Y. Jiang, L. Liu, and J. J. Steinle, “Compound 49b regulates ZO1 and occludin levels in human retinal endothelial cells and in mouse retinal vasculature," Investigative Ophthalmology \& Visual Science, vol. 58, no. 1, pp. 185-189, 2017.

[22] S. Saker, E. A. Stewart, A. C. Browning, C. L. Allen, and W. M. Amoaku, "The effect of hyperglycaemia on permeability and the expression of junctional complex molecules in human retinal and choroidal endothelial cells," Experimental Eye Research, vol. 121, pp. 161-167, 2014.

[23] H. Yamada, E. Yamada, A. Higuchi, and M. Matsumura, "Retinal neovascularisation without ischaemia in the spontaneously diabetic Torii rat," Diabetologia, vol. 48, no. 8, pp. 1663-1668, 2005.
[24] M. Matsuoka, N. Ogata, K. Minamino, A. Higuchi, and M. Matsumura, "High levels of pigment epithelium-derived factor in the retina of a rat model of type 2 diabetes," Experimental Eye Research, vol. 82, no. 1, pp. 172-178, 2006.

[25] F. Bandello, R. Lattanzio, I. Zucchiatti, and C. Del Turco, "Pathophysiology and treatment of diabetic retinopathy," Acta Diabetologica, vol. 50, no. 1, pp. 1-20, 2013.

[26] S. Vujosevic and R. Simó, "Local and systemic inflammatory biomarkers of diabetic retinopathy: an integrative approach," Investigative Ophthalmology \& Visual Science, vol. 58, no. 6, pp. BIO68-BIO75, 2017.

[27] Y. Yafai, I. Iandiev, J. Lange et al., "Basic fibroblast growth factor contributes to a shift in the angioregulatory activity of retinal glial (Müller) cells," PLoS One, vol. 8, no. 7, article e68773, 2013.

[28] J. H. Kim, J. H. Kim, Y. S. Yu, C. S. Cho, and K. W. Kim, "Blockade of angiotensin II attenuates VEGF-mediated blood-retinal barrier breakdown in diabetic retinopathy," Journal of Cerebral Blood Flow \& Metabolism, vol. 29, no. 3, pp. 621-628, 2009.

[29] C. Hernández, M. Dal Monte, R. Simó, and G. Casini, "Neuroprotection as a therapeutic target for diabetic retinopathy," Journal of Diabetes Research, vol. 2016, Article ID 9508541, 18 pages, 2016.

[30] M. Fukuda, Y. Nakanishi, M. Fuse et al., "Altered expression of aquaporins 1 and 4 coincides with neurodegenerative events in retinas of spontaneously diabetic Torii rats," Experimental Eye Research, vol. 90, no. 1, pp. 17-25, 2010.

[31] F. Toyoda, Y. Tanaka, A. Ota et al., "Effect of ranirestat, a new aldose reductase inhibitor, on diabetic retinopathy in SDT rats," Journal of Diabetes Research, vol. 2014, Article ID 672590, 7 pages, 2014.

[32] C. Motta, G. Lupo, D. Rusciano et al., "Molecular mechanisms mediating antiangiogenic action of the urokinase receptorderived peptide UPARANT in human retinal endothelial cells," Investigative Ophthalmology \& Visual Science, vol. 57, no. 13, pp. 5723-5735, 2016.

[33] M. Fukumoto, S. Takai, E. Ishizaki et al., "Involvement of angiotensin II-dependent vascular endothelial growth factor gene expression via NADPH oxidase in the retina in a type 2 diabetic rat model," Current Eye Research, vol. 33, no. 10, pp. 885-891, 2008.

[34] J. Ideno, H. Mizukami, A. Kakehashi et al., "Prevention of diabetic retinopathy by intraocular soluble flt- 1 gene transfer in a spontaneously diabetic rat model," International Journal of Molecular Medicine, vol. 19, no. 1, pp. 75-79, 2007.

[35] T. Sugiyama, T. Okuno, M. Fukuhara et al., "Angiotensin II receptor blocker inhibits abnormal accumulation of advanced glycation end products and retinal damage in a rat model of type 2 diabetes," Experimental Eye Research, vol. 85, no. 3, pp. 406-412, 2007.

[36] P. F. Kador, M. Wyman, and P. J. Oates, “Aldose reductase, ocular diabetic complications and the development of topical Kinostat ${ }^{\circledast}, "$ Progress in Retinal and Eye Research, vol. 54, pp. 1-29, 2016.

[37] M. H. Petri, A. Laguna-Fernández, M. Gonzalez-Diez, G. Paulsson-Berne, G. K. Hansson, and M. Bäck, "The role of the FPR2/ALX receptor in atherosclerosis development and plaque stability," Cardiovascular Research, vol. 105, no. 1, pp. $65-74,2015$. 
[38] M. Iskar, M. Campillos, M. Kuhn, L. J. Jensen, V. van Noort, and P. Bork, "Drug-induced regulation of target expression," PLoS Computational Biology, vol. 6, no. 9, article e1000925, 2010.

[39] M. Lohse, "Molecular mechanisms of membrane receptor desensitization," Biochimica et Biophysica Acta (BBA) - Molecular Cell Research, vol. 1179, no. 2, pp. 171-188, 1993.

[40] M. G. Eason, H. Kurose, B. D. Holt, J. R. Raymond, and S. B. Liggett, "Simultaneous coupling of alpha 2-adrenergic receptors to two G-proteins with opposing effects. Subtypeselective coupling of alpha $2 \mathrm{C} 10$, alpha $2 \mathrm{C} 4$, and alpha $2 \mathrm{C} 2$ adrenergic receptors to $\mathrm{Gi}$ and Gs," Journal of Biological Chemistry, vol. 267, no. 22, pp. 15795-15801, 1992.

[41] I. Arbeláez-Quintero and M. Palacios, “To use or not to use metformin in cerebral ischemia: a review of the application of metformin in stroke rodents," Stroke Research and Treatment, vol. 2017, Article ID 9756429, 13 pages, 2017.

[42] X. Liu, F. Ye, H. Xiong et al., "IL- $1 \beta$ induces IL-6 production in retinal Müller cells predominantly through the activation of P38 MAPK/NF- $k$ B signaling pathway," Experimental Cell Research, vol. 331, no. 1, pp. 223-231, 2015.

[43] R. de Hoz, B. Rojas, A. I. Ramírez et al., "Retinal macroglial responses in health and disease," BioMed Reserach International, vol. 2016, article 2954721, 13 pages, 2016.

[44] S. Rezzola, M. Corsini, P. Chiodelli et al., "Inflammation and $\mathrm{N}$-formyl peptide receptors mediate the angiogenic activity of human vitreous humour in proliferative diabetic retinopathy," Diabetologia, vol. 60, no. 4, pp. 719-728, 2017.

[45] S. Boccella, E. Panza, L. Lista et al., "Preclinical evaluation of the urokinase receptor-derived peptide UPARANT as an anti-inflammatory drug," Inflammation Research, vol. 66, no. 8, pp. 701-709, 2017.

[46] A. Das, P. G. McGuire, and F. Monickaraj, "Novel pharmacotherapies in diabetic retinopathy: current status and what's in the horizon?," Indian Journal of Ophthalmology, vol. 64, no. 1, pp. 4-13, 2016. 


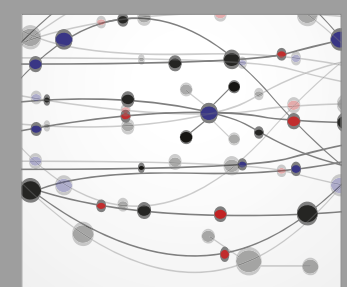

The Scientific World Journal
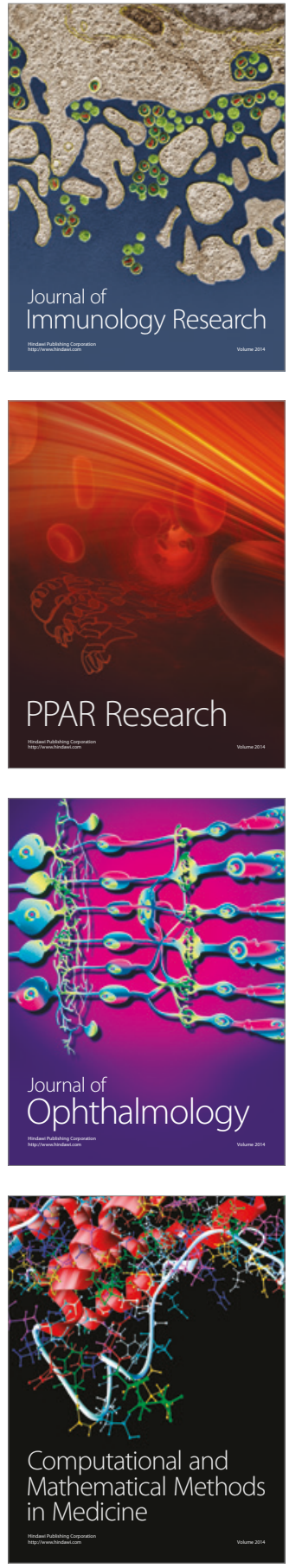

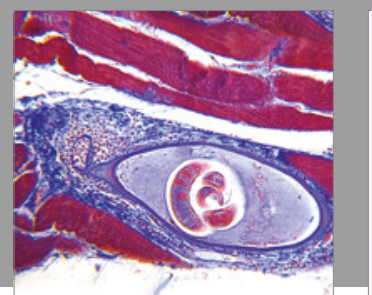

Gastroenterology Research and Practice
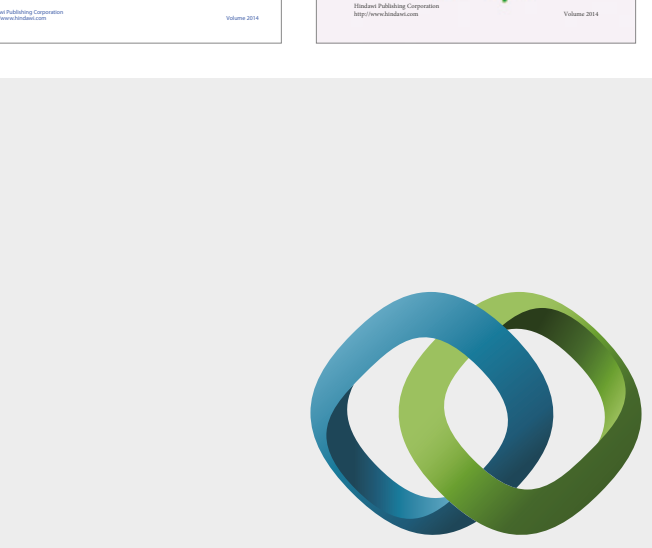

\section{Hindawi}

Submit your manuscripts at

https://www.hindawi.com
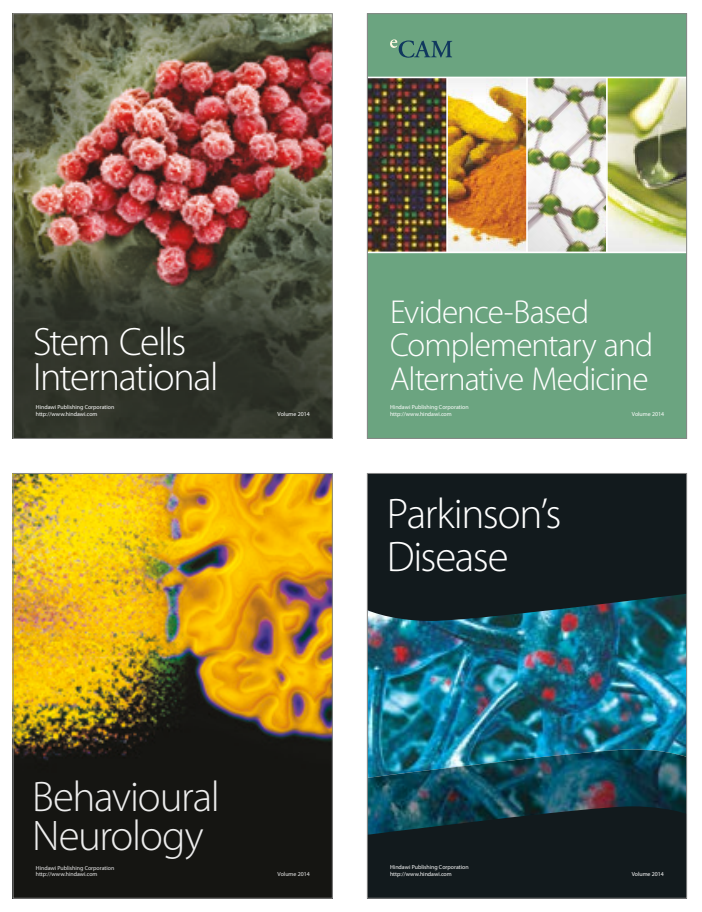
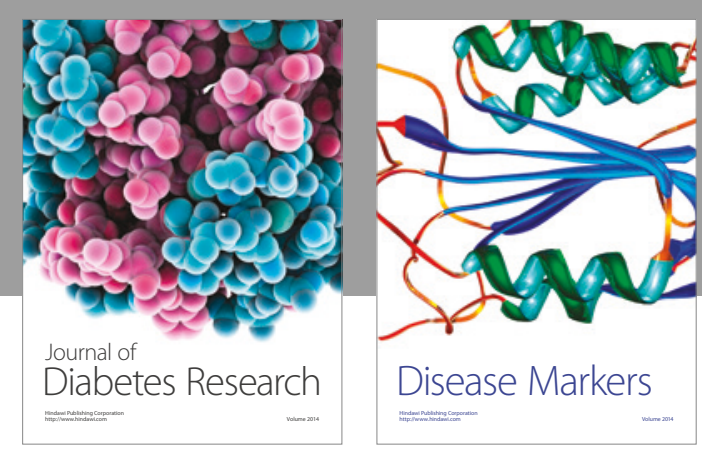

Disease Markers
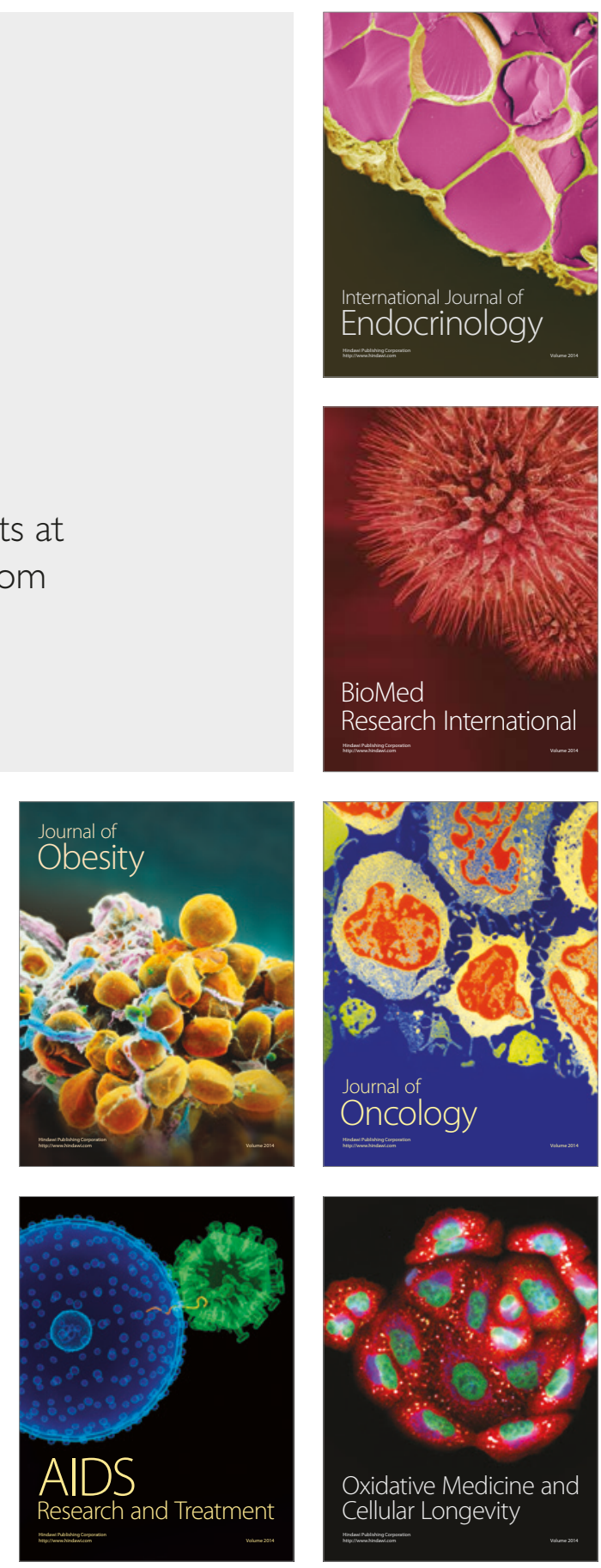